\title{
Regressão linear com medidas censuradas
}

\author{
Marcel Frederico de Lima Taga
}

DISSERTAÇÃO APRESENTADA AO INSTITUTO DE MATEMÁTICA E ESTATÍSTICA

DA UNIVERSIDADE DE SÃO PAULO

PARA OBTENÇÃO DO TÍTULO DE MESTRE EM CIÊNCIAS

Programa: Estatística

Orientador: Prof. Dr. Julio da Motta Singer

São Paulo, dezembro de 2008 



\section{Regressão linear com medidas censuradas}

Este exemplar corresponde à redação final da dissertação devidamente corrigida e defendida por Marcel Frederico de Lima Taga e aprovada pela Comissão Julgadora.

Comissão Julgadora:

- Prof. Dr. Julio da Motta Singer (orientador) - IME - USP

- Prof. Dr. Clóvis de Araújo Peres - IME - USP

- Prof. Dr. Juvêncio Santos Nobre - UFC 

Aos meus pais Maria Silvia e Eulázio, a minha avó Tereza e em memória dos meus avôs Abel e Hiromi e da minha avó Iracema. 



\section{Agradecimentos}

Gostaria de agradecer a todas as pessoas que de alguma maneira contribuíram para que eu pudesse concluir este projeto. Especialmente:

Ao professor Julio da Motta Singer pela paciência, apoio, incentivo e pela leitura criteriosa de inúmeras versões deste trabalho até em horários fora do expediente.

Ao professor Clóvis de Araújo Peres, por ter me ensinado o que é Estatística Aplicada e por seu apoio constante para o sucesso da minha vida pessoal e profissional.

Ao professor Juvêncio Santos Nobre pelos comentários e sugestões.

À Tatiana Terabayashi Melhado, pela grande ajuda durante estes anos de Mestrado e ao Daniel Kashiwamura Scheffer por ter sido como um irmão para mim, além da ajuda nesta reta final do curso.

À Otília da Costa Paulon pela constante preocupação para que eu pudesse sempre progredir pessoal e profissionalmente.

À professora Viviana Giampaoli por ter me ajudado a realizar os seminários relativos a este trabalho, sem isto, dificilmente eu conseguiria realizar este projeto.

Aos meus pais Maria Silvia de Lima Taga e Eulázio de Lima Taga pelo apoio e carinho que sempre me deram.

Ao meu irmão Marcio Luiz Lima Taga pela preocupação, apoio e incentivo durante todos estes anos.

À minha cunhada Josilene Luciene Duarte e à Duda pela paciência e pela ajuda na leitura de inúmeras versões, e pelo esclarecimento de inúmeras dúvidas dos dados utilizados neste trabalho. 
Ao João Henrique Nogueira Pinto por ter possibilitado alternativas, como meu chefe no HRAC, para que eu pudesse concluir este projeto. Sem isto, também seria difícil a conclusão desta dissertação.

Ao amigo Saulo José Garcia e às amigas Alzira Aparecida Cantro Quirino dos Santos, Silvia Mara Desan Merli, Eleide Miriam Bim Bahia e Rosibel Cristina de Paula Rasi pelo apoio constante tanto pessoal como profissional.

À Ana A. Gomes Grigolli pela ajuda com a aquisição das referências bibliográficas, sempre de forma rápida e criteriosa.

À Kátia de Freitas Alvarenga pelo apoio profissional e pessoal e pelo carinho.

Ao professor Otávio Lins por valorizar meu trabalho.

À Luzia Maria Pozzobom Ventura e à sua família pelo apoio, carinho e orações.

A profesora Mary Lou Thompson pela atenção e esclarecimento de algumas dúvidas.

À Regina Helena Russo por ter me dado a oportunidade de conhecer o professor Clóvis e por me ajudar profissionalmente.

À Marília Sandoval Pássaro e sua família pelo apoio e pelo carinho.

Ao Walter Duarte, à Ana Maria Silva Duarte, à Janaína Luciane Duarte, a Cleuza Maria de Andrade Ussuna, ao Guilherme de Andrade de Ussuna e ao Daniel de Andrade Ussuna pelo companheirismo.

Aos amigos Marcelo Hiroshi Ogava, Ângela Tavares Paes, Gianni Yanaguibashi, Mayra Ivanoff Lora, Frederico Zanqueta Poleto, Rodrigo Vianna Rocha, Dalton Santos Pinheiro, Roberta Okura, Milena Reis, Kelsy Catherina Nema Areco e Gizelton Pereira Alencar pela ajuda durante o curso de mestrado.

Ao Fábio Tadeu Montesano pela ajuda durante o tempo em que trabalhamos juntos.

Ao Rodrigo Futata Fujii pela valorização profissional.

À Ana Dolores e ao Juan pelo incentivo.

À Leni Pereira da Silva por organizar a minha bagunça sem que eu ficasse perdido com as coisas organizadas e pela torcida para que tudo desse certo.

Aos amigos de Bauru, Daniel Imaisumi, Fábio Imaisumi, Vinicius Bim, Mar- 
celo José dos Santos, Héverton Vicente, José Ernesto Campesi, Eduardo Varallo, Alexandre Viegas e George Vidal pelas palavras de apoio nos momentos de desânimo.

Ao meu grande amigo Walter Esbaille pela ajuda nos momentos difíceis.

A todos os professores do IME que me deram a oportunidade de progredir, em especial à professora Zara Issa Abud que conseguiu resgatar a minha vontade de estudar e ao professor Marcos Nascimento Magalhães pela orientação nos primeiros anos da minha graduação.

Ao meu amor Cibele de Andrade Ussuna pelo apoio, companheirismo e principalmente pela compreensão e paciência.

A Deus por ter me dado a oportunidade de conhecer tantas pessoas especiais durante estes anos de estudo e por ter me iluminado para realização deste projeto. 

"Provações te buscaram.

Dificuldades te agitam.

Tudo parece noite ao redor de teus passos.

Não te detenhas, no entanto, a fim de medir as sombras.

Prossegue trabalhando e não te afastes da paciência.

Por nada te desesperes.

Dá tempo a Deus para que Deus te acenda nova luz."

Emmanuel 



\section{Resumo}

Consideramos um modelo de regressão linear simples, em que tanto a variável resposta como a independente estão sujeitas a censura intervalar. Como motivação utilizamos um estudo em que o objetivo é avaliar a possibilidade de previsão dos resultados de um exame audiológico comportamental a partir dos resultados de um exame audiológico eletrofisiológico. Calculamos intervalos de previsão para a variável resposta, analisamos o comportamento dos estimadores de máxima verossimilhança obtidos sob o modelo proposto e comparamos seu desempenho com aquele de estimadores obtidos de um modelo de regressão linear simples usual, no qual a censura dos dados é desconsiderada.

Palavras-chave: Regressão linear, censura intervalar, intervalo de previsão. 



\begin{abstract}
We consider a simple linear regression model in which both variables are interval censored. To motivate the problem we use data from an audiometric study designed to evaluate the possibility of prediction of behavioral thresholds from physiological thresholds. We develop prediction intervals for the response variable, obtain the maximum likelihood estimators of the proposed model and compare their performance with that of estimators obtained under ordinary linear regression models.
\end{abstract}

Key words: Linear regression, interval censoring, prediction interval. 



\section{Sumário}

1 Introdução 1

2 Modelo e função de verossimilhança 15

3 Ajuste dos modelos $\quad 17$

4 Intervalos de previsão $\quad 33$

5 Simulações $\quad 37$

6 Discussão 45

A Método de Newton-Raphson $\quad 53$

B Expressões para o vetor score e matriz Hessiana de $l(\theta)$

$\begin{array}{lr}\text { Referências Bibliográficas } & 65\end{array}$ 



\section{Capítulo 1}

\section{Introdução}

O desenvolvimento normal da linguagem e da fala depende, entre outros fatores, da integridade do sistema auditivo. Ao nascer, o córtex auditivo do feto apresenta trilhões de conexões neuronais, que se não forem estimuladas, como no caso da surdez, entram em um processo de definhamento (Northern e Downs, 2002). Por isso, existe a necessidade de diagnosticar a deficiência auditiva logo nos primeiros meses de vida, e, se necessário, dar início ao processo de reabilitação.

Para o diagnóstico da deficiência auditiva podem ser utilizados procedimentos comportamentais, eletroacústicos e eletrofisiológicos. Procedimentos comportamentais, como a Audiometria Tonal Liminar (ATL) dependem da resposta consciente do indivíduo para uma tarefa solicitada. Os procedimentos eletroacústicos e eletrofisiológicos não dependem de uma resposta do paciente e podem ser realizados com o indivíduo em estado de sono ou sedado.

O procedimento considerado como o padrão áureo para medir o limiar auditivo (menor intensidade de um som que o indivíduo é capaz de ouvir) é a ATL. Em recém-nascidos e crianças que ainda não conseguem realizar este exame, o limiar auditivo pode ser estimado pelo limiar eletrofisiológico (menor intensidade de um som capaz de gerar uma atividade elétrica captável nas estruturas neuronais) por meio do exame conhecido como Resposta Auditiva de Estado Estável (RAEE).

Em um estudo realizado por Duarte (2007), o objetivo era verificar a aplicabilidade do RAEE para prever os limiares auditivos obtidos por ATL. Nesse 
estudo, os dois exames (ATL e RAEE) foram aplicados em 48 voluntários, com idade entre 7 e 30 anos, atendidos pela Clínica de Fonoaudiologia da Faculdade de Odontologia de Bauru (FOB) e pelo Centro de Pesquisas Audiológicas do Hospital de Reabilitação de Anomalias Craniofaciais (CPA-HRAC), ambas instituições da Universidade de São Paulo (USP). Parte dos dados deste estudo encontra-se na Tabela 1.1. Estes valores foram obtidos por ATL e por RAEE para um tom com freqüência de 4,0 kHz e foram medidos em decibel Nível de Audição (dBNA ${ }^{1}$ ).

Tabela 1.1: Valores utilizados na clínica como limiar auditivo e eletrofisiológico (dBNA) e intervalo de censura destes valores obtidos por ATL e RAEE da orelha direita (OD) para um tom com freqüência de $4,0 \mathrm{kHz}$

\begin{tabular}{cccccc}
\hline Ind & ATL & RAEE & Ind & ATL & RAEE \\
\hline 1 & $75(70 ; 75]$ & $75(70 ; 75]$ & 25 & $45(40 ; 45]$ & $60(55 ; 60]$ \\
2 & $25(20 ; 25]$ & $30(25 ; 30]$ & 26 & $55(50 ; 55]$ & $115(110 ; 115]$ \\
3 & $5(0 ; 5]$ & $40(35 ; 40]$ & 27 & $85(80 ; 85]$ & $100(95 ; 100]$ \\
4 & $20(15 ; 20]$ & $40(35 ; 40]$ & 28 & $70(65 ; 70]$ & $90(85 ; 90]$ \\
5 & $40(35 ; 40]$ & $35(30 ; 35]$ & 29 & $105(100 ; 105]$ & $115(115 ;+\infty)$ \\
6 & $40(35 ; 40]$ & $70(65 ; 70]$ & 30 & $115(115 ;+\infty)$ & $118(118 ;+\infty)$ \\
7 & $55(50 ; 55]$ & $80(75 ; 80]$ & 31 & $100(95 ; 100]$ & $115(110 ; 115]$ \\
8 & $30(25 ; 30]$ & $40(35 ; 40]$ & 32 & $75(70 ; 75]$ & $70(65 ; 70]$ \\
9 & $45(40 ; 45]$ & $80(75 ; 80]$ & 33 & $75(70 ; 75]$ & $100(95 ; 100]$ \\
10 & $25(20 ; 25]$ & $35(30 ; 35]$ & 34 & $95(90 ; 95]$ & $110(105 ; 110]$ \\
11 & $70(65 ; 70]$ & $80(75 ; 80]$ & 35 & $80(75 ; 80]$ & $95(90 ; 95]$ \\
12 & $60(55 ; 60]$ & $70(65 ; 70]$ & 36 & $115(110 ; 115]$ & $110(105 ; 110]$ \\
13 & $50(45 ; 50]$ & $60(55 ; 60]$ & 37 & $90(85 ; 90]$ & $118(118 ;+\infty)$ \\
\hline & & & & & continua
\end{tabular}

\footnotetext{
${ }^{1} \mathrm{O}$ termo decibel é utilizado expressar uma quantidade física qualquer em relação a um valor de referência. Em particular, a mensuração da intensidade sonora pode ser feita quantificando-se a relação entre a pressão sonora de um determinado tom e um valor de pressão sonora de referência. Quando esta intensidade é dada por $I_{d B N A}=20 \log \left(P / P_{0}\right)$, sendo $P$ o valor de pressão sonora do tom e $P_{0}$ o valor de pressão sonora mínima para que um tom de determinada frequiência seja audível em indivíduos normais, dizemos que a intensidade do tom foi medida em dBNA (Russo, 1999). Exemplo: para um tom de $1000 \mathrm{~Hz}, P_{0}=20 \mu P a$, logo, se o limiar auditivo do indivíduo é de $0 \mathrm{dBNA}$, a pressão sonora mínima necessária para que inidvíduo escute o som é de $20 \mu P a$.
} 


\begin{tabular}{cccccc}
\hline Ind & ATL & RAEE & Ind & ATL & RAEE \\
\hline 14 & $50(45 ; 50]$ & $60(55 ; 60]$ & 38 & $115(110 ; 115]$ & $118(118 ;+\infty)$ \\
15 & $65(60 ; 65]$ & $70(65 ; 70]$ & 39 & $110(105 ; 110]$ & $108(103 ; 108]$ \\
16 & $45(40 ; 45]$ & $60(55 ; 60]$ & 40 & $110(105 ; 110]$ & $100(95 ; 100]$ \\
17 & $60(55 ; 60]$ & $100(95 ; 100]$ & 41 & $0(-\infty ; 0]$ & $10(5 ; 10]$ \\
18 & $75(70 ; 75]$ & $85(80 ; 85]$ & 42 & $115(115 ;+\infty)$ & $118(118 ;+\infty)$ \\
19 & $115(115 ;+\infty)$ & $118(113 ; 118]$ & 43 & $115(110 ; 115]$ & $110(105 ; 110]$ \\
20 & $70(65 ; 70]$ & $110(105 ; 110]$ & 44 & $115(110 ; 115]$ & $118(118 ;+\infty)$ \\
21 & $50(45 ; 50]$ & $70(65 ; 70]$ & 45 & $110(105 ; 110]$ & $115(110 ; 115]$ \\
22 & $55(50 ; 55]$ & $65(60 ; 65]$ & 46 & $115(115 ;+\infty)$ & $118(118 ;+\infty)$ \\
23 & $70(65 ; 70]$ & $100(100 ;+\infty)$ & 47 & $115(115 ;+\infty)$ & $118(113 ; 118]$ \\
24 & $70(65 ; 70]$ & $80(75 ; 80]$ & 48 & $115(115 ;+\infty)$ & $118(118 ;+\infty)$ \\
\hline
\end{tabular}

Nesse estudo, foram avaliados os limiares auditivos e eletrofisiológicos das duas orelhas para tons de 0,5, 1,0,2,0 e 4,0 kHz. O primeiro exame realizado foi o ATL. Nesse exame, as medidas dos quatro tons foram obtidas primeiramente em uma orelha e depois na outra. A ordem em que os tons foram aplicados foi: $0,5,1,0,2,0$, e 4,0 kHz. A escolha da primeira orelha foi realizada de forma sequencial, alternando-se os lados de acordo com a admissão dos pacientes. Para o RAEE, valores de até $80 \mathrm{dBNA}$ foram testados simultaneamente para os quatro tons e para as duas orelhas (Lins e Picton, 1995). Acima deste valor, as medidas dos limiares restantes foram feitas primeiro em uma orelha e depois na outra, seguindo a mesma ordem de lado estabelecida para o exame de ATL e utilizando a ordem de: $0,5,4,0,1,0$ e 2,0 kHz, para teste dos tons.

Para determinar o limiar auditivo ou o limiar eletrofisiológico, utiliza-se o seguinte procedimento: a partir de um determinado valor de intensidade, estipulado por especialistas, um estímulo sonoro (um tom de determinada freqüência) é enviado ao indivíduo através de fones. Se houver resposta (comportamental, no caso da ATL ou eletrofisiológica, no caso da RAEE), a intensidade do tom é diminuída de 10 em 10 dBNA. Quando não há mais resposta, a intensidade do tom é aumentada de 5 em 5 dBNA até que ocorra resposta novamente e essa intensidade de tom é utilizada na clínica como o limiar auditivo, no caso da ATL e como o limiar 
eletrofisiológico, no caso da RAEE. Quando não há resposta ao estímulo inicial, a intensidade é aumentada de 10 em 10 dBNA até que haja resposta e depois diminuída de 5dBNA para determinar o valor que será utilizado como limiar.

Utilizando-se este procedimento, sabe-se apenas que o valor do limiar pertence a um intervalo de amplitude de 5 dBNA. Nesta situação, dizemos que o valor do limiar está sujeito à censura intervalar (Klein e Moeschberger, 1997). Na Tabela 1.1 encontram-se tanto os valores obtidos através deste procedimento, quanto os intervalos de censura correspondentes. O indivíduo 1, por exemplo, apresenta limiar auditivo e eletrofisiológico, maior que 70 dBNA e menor ou igual a 75 dBNA.

$\mathrm{Na}$ mesma tabela, é possível verificar a existência de valores em que a única informação disponível é a de que o valor do limiar auditivo é menor ou igual a 0 . Também encontramos casos em que a única informação disponível é a de que o valor do limiar auditivo ou eletrofisiológico é maior que alguma constante. Isto pode ocorrer porque os valores de intensidade utilizados pelos dois exames estão restritos a valores mínimos e máximos. A intensidade mínima testada para todos os tons nos dois exames foi de $0 \mathrm{dBNA}$. As intensidades máximas utilizadas para ATL foram: 115, 120, 115 e 115 dBNA para os tons de 0,5, 1,0, 2,0 e 4,0 kHz, respectivamente. Para a RAEE, os valores máximos utilizados foram: 117, 121, 119 e 118 dBNA para os tons de 0,5, 1,0,2,0 e 4,0 kHz, respectivamente. Utilizando a denominação dada por Klein e Moeschberger (1997), dizemos que existem valores censurados à esquerda (valor de ATL do indivíduo 41, por exemplo) e valores censurados à direita (valores de ATL e RAEE do indivíduo 48, por exemplo).

No exame de RAEE também pode ocorrer censura à direita, porque, para cada indivíduo, acima do limiar auditivo, existe um valor de intensidade desconhecido que pode causar desconforto. Se não houver resposta eletrofisiológica até esse valor, é necessário interromper o exame de RAEE para não causar danos ao sistema auditivo; foi o que ocorreu com o indivíduo 23, por exemplo. Nesse caso, a única informação disponível é que o limiar obtido por RAEE é maior que 100 dBNA. 
Nas Figuras 1.1 e 1.2 encontram-se os gráficos de dispersão entre os valores utilizados na clínica como limiares, obtidos por ATL e por RAEE para cada orelha e freqüência, além de um segmento de reta com inclinação de $45^{\circ}$. Os gráficos sugerem a existência de associação linear entre os valores obtidos por ATL e por RAEE. Também é possível notar que os valores obtidos por RAEE, em geral, são maiores do que aqueles obtidos por ATL.

O principal objetivo deste trabalho é prever o limiar auditivo obtido por ATL a partir do limiar eletrofisiológico obtido por RAEE, e determinar e o erro de previsão. Se nos restringíssimos a análise dos dados de uma orelha e uma freqüência, e, se os limiares dos dois exames não fossem censurados, poderíamos dizer que o objetivo deste estudo é ajustar um modelo de regressão linear simples:

$$
Y_{i}=\alpha+\beta X_{i}+\epsilon_{i}
$$

em que $\alpha$ e $\beta$ são parâmetros do modelo, $Y_{i}$ representa o limiar auditivo do iésimo indivíduo obtido por ATL, $X_{i}$ representa o limiar eletrofisiológico do iésimo indivíduo obtido por RAEE, $\epsilon_{i}$ são variáveis aleatórias independentes, com distribuição $N\left(0, \sigma^{2}\right), i=1, . ., n$, e $n$ é o tamanho da amostra

Para prever o limiar auditivo obtido por ATL utilizando o limiar eletrofisiológico obtido por RAEE, bastaria utilizar métodos usuais para regressão linear simples (Neter et al., 1996). Quando a variável resposta ou a variável explicativa estão sujeitas a censura, este modelo pode gerar estimativas enviesadas.

Na literatura de modelos de análise de sobrevivência, o termo censura à direita do Tipo I é utilizado quando o evento de interesse só é observado se ocorrer antes de um tempo predeterminado. Por exemplo, para estudar o tempo de sobrevida de $N$ lâmpadas, um pesquisador aguarda até um tempo predeterminado $(T)$ e conta o número de lâmpadas queimadas. O tempo de sobrevida do i-ésimo indivíduo $\left(T_{i}\right)$ é dado pelo mínimo $\left(T_{i}, T\right)$. A diferença entre a censura do Tipo I e a censura do Tipo II, é que no segundo caso, não existe um tempo predeterminado para encerrar o estudo. O término só ocorre quando um número predeterminado de eventos ocorrer. Utilizando o mesmo exemplo das lâmpadas, o pesquisador só encerra o estudo quando um número predeterminado, $n$, das $N$ lâmpadas queimarem. Então, 
Figura 1.1: Gráficos de dispersão entre os valores utilizados na clínica como limiares auditivos obtidos por ATL vs valores utilizados na clínica como limiares eletrofisiológicos obtidos por RAEE para cada orelha e freqüências de 0,5 e 1,0 kHz.

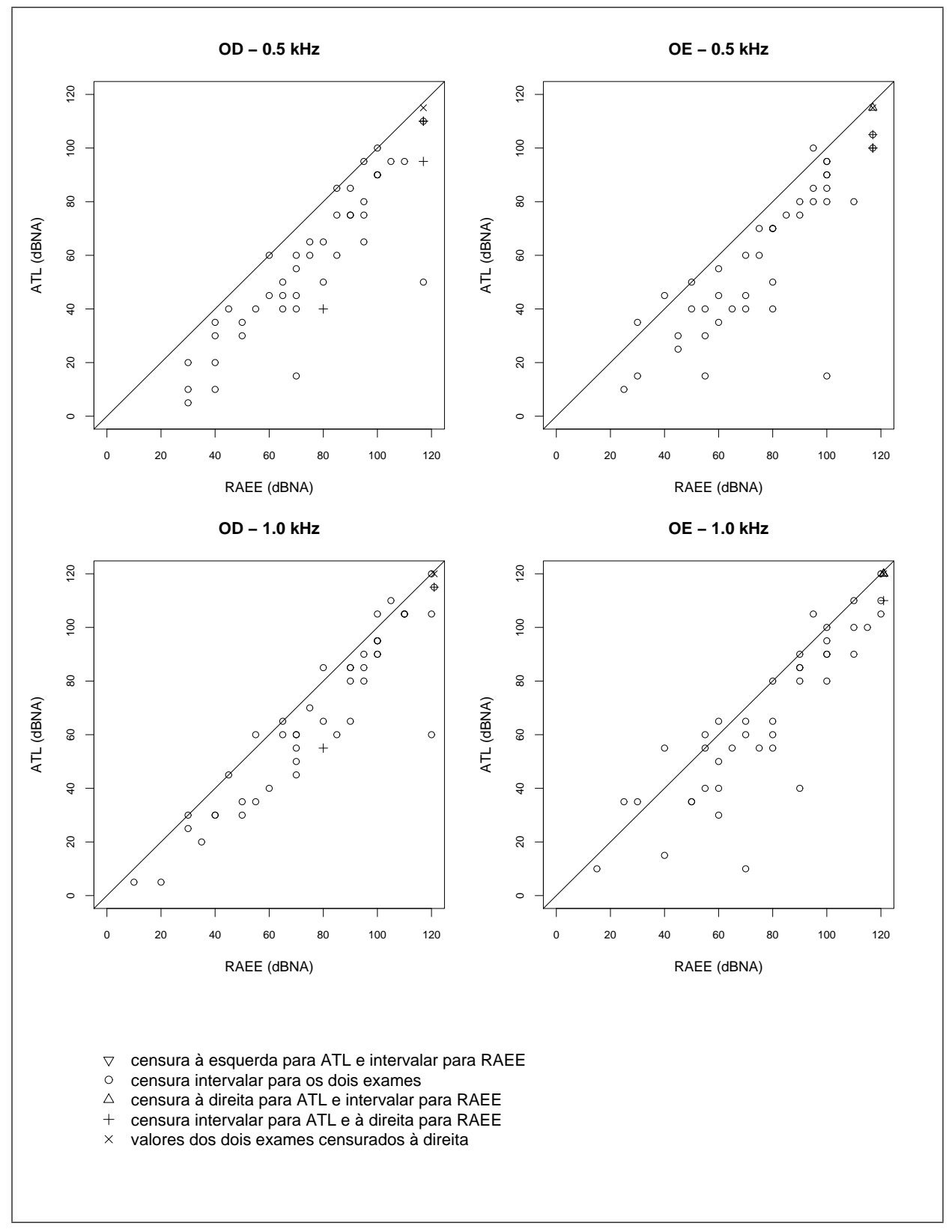


Figura 1.2: Gráficos de dispersão entre os valores utilizados na clínica como limiares auditivos obtidos por ATL vs valores utilizados na clínica como limiares eletrofisiológicos obtidos por RAEE para cada orelha e frequiências de 2,0 e 4,0 kHz.

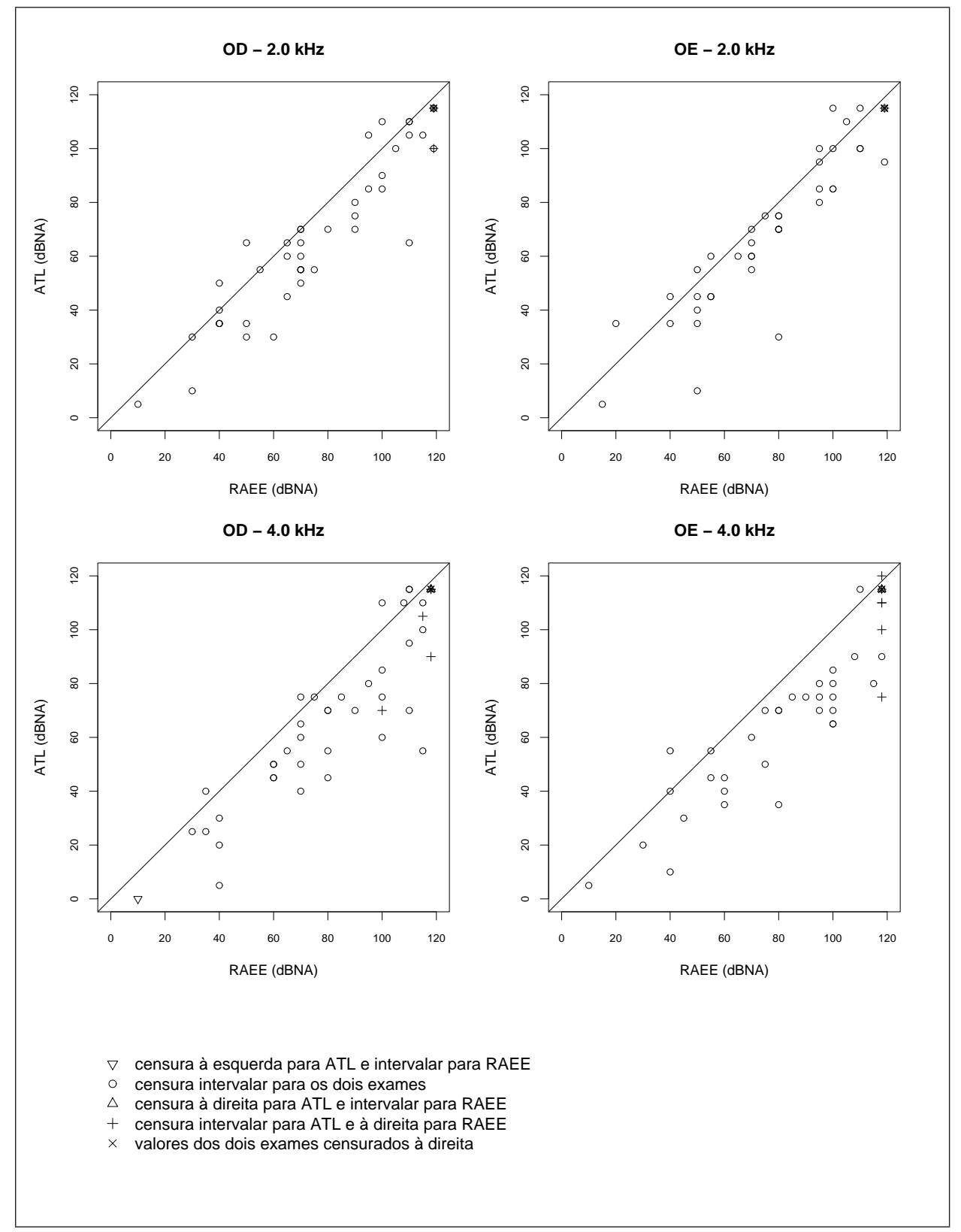


quando a censura é do Tipo II, é possível saber a priori, o número de valores censurados.

Para um modelo de regressão linear simples, em que a variável resposta está sujeita a censura do Tipo I à direita, a função de verossimilhança é:

$$
L\left(\alpha, \beta, \sigma^{2} ; \mathbf{x}, \mathbf{y}\right)=\prod_{i=1}^{n}\left[f\left(y_{i} ; x_{i}, \alpha, \beta, \sigma^{2}\right)\right]^{\delta_{i}}\left[\int_{L C S}^{+\infty} f\left(z ; x_{i}, \alpha, \beta, \sigma^{2}\right) d z\right]^{\left(1-\delta_{i}\right)}
$$

em que

$$
\begin{gathered}
f\left(y_{i} ; x_{i}, \alpha, \beta, \sigma^{2}\right)=\frac{1}{\sqrt{2 \pi \sigma^{2}}} \exp \left(-\frac{\left(y_{i}-\left(\alpha+\beta x_{i}\right)\right)}{2 \sigma^{2}}\right), \\
\delta_{i}= \begin{cases}0, & \text { quando } y_{i}>C \\
1, & \text { quando } y_{i} \leq C,\end{cases}
\end{gathered}
$$

$\mathrm{C}$ é o ponto de corte de censura, $\mathbf{y}=\left(y_{1}, y_{2}, . ., y_{n}\right)$ e $\mathbf{x}=\left(x_{1}, x_{2}, \ldots, x_{p}\right)$ são os valores observados de $\mathbf{Y}=\left(Y_{1}, Y_{2}, . ., Y_{n}\right)$ e $\mathbf{X}=\left(X_{1}, X_{2}, \ldots, X_{p}\right)$.

Glasser (1965) propôs estimadores de máxima verossimilhança para os parâmetros de um modelo de regressão linear múltiplo, em que a variável resposta tem distribuição normal e está sujeita à censura do Tipo I à direita. Como exemplo, ele considerou o ajuste de um modelo de regressão linear, entre o logaritmo natural do tempo de sobrevivência de indivíduos com determinada doença e duas variáveis independentes.

Problemas similares, em que a variável resposta está sujeita a censura também são encontrados na área ambiental. É comum encontrar situações, em que o objetivo é estudar a associação entre a concentração de determinado contaminante (variável resposta) e outras variáveis independentes. A censura ocorre porque não é possível mensurar a concentração do contaminante abaixo de um limite de detecção. Para resolver este problema, (Haas e Jacangelo, 1993) também propuseram uma solução por máxima verossimilhança. Utilizando simulações, compararam as estimativas obtidas por máxima verossimilhança com as estimativas obtidas pelo método de mínimos quadrados após a eliminação das respostas censuradas. Para níveis de censura entre $25 \%$ e $75 \%$, as estimativas obtidas por máxima verossimi- 
lhança apresentaram um viés menor e uma variação menor quando a distribuição assumida para os erros era normal. Nos casos em que a distribuição assumida para os erros era lognormal, as estimativas apresentaram praticamente o mesmo grau de viés e variabilidade.

$\mathrm{Na}$ área de Econometria, Tobin (1958) propôs o uso de estimativas obtidas por máxima verossimilhança para um modelo semelhante a uma regressão linear múltipla em que a variável resposta está sujeita a censura à esquerda. O modelo adotado foi:

$$
y_{i}=\left\{\begin{array}{l}
\boldsymbol{\beta}^{t} \mathbf{x}_{\mathbf{i}}+\epsilon_{i}, \text { se } \boldsymbol{\beta}^{t} \mathbf{x}_{\mathbf{i}}+\epsilon_{i}>0 \\
0, \text { se } \boldsymbol{\beta}^{t} \mathbf{x}_{\mathbf{i}}+\epsilon_{i} \leq 0
\end{array}\right.
$$

em que $\boldsymbol{\beta}=\left(\beta_{0}, \beta_{1}, \ldots, \beta_{p}\right)^{t}$ é um vetor de parâmetros, $\mathbf{x}_{\mathbf{i}}=\left(x_{i 1}, x_{i 2}, \ldots, x_{i p}\right)^{t}$ é um vetor com valores das variáveis explicativas para o i-ésimo indivíduo e $\epsilon_{i} \sim$ $N\left(0, \sigma^{2}\right)$ são variáveis aleatórias independentes que representam erros aleatórios.

Amemiya (1973) aprimorou e estendeu esse modelo, provando que os estimadores de máxima verossimilhança são consistentes e que sua distribuição é assintoticamente normal. A diferença entre esse modelo e os anteriores, é que a variável resposta não é uma variável censurada, mas sim limitada ao valor 0 . Nesse modelo atribui-se uma probabilidade positiva à resposta nula. Pode-se dizer que é um modelo de dados censurados, adaptado para o caso de uma variável limitada. Ele pode ser utilizado, por exemplo, quando a variável resposta é o gasto familiar com algum bem durável num determinado período.

Além do método de máxima verossimilhança, outros métodos foram propostos para o caso de um modelo de regressão linear, em que o erro tem distribuição normal e a variável resposta está sujeita a censura do Tipo I à direita. O metodo de Schmee e Hahn (1979) consiste no uso iterativo do método de mínimos quadrados, substituindo-se os valores censurados pelos valores esperados até que ocorra convergência. Para um modelo de regressão linear simples, em que a variável está sujeita à censura do Tipo I à direita com limite de censura dado por $\mathrm{C}$, o valor esperado quando $Y_{i}>C$ é dado por (Schneider, 1986):

$$
\mu_{y_{i}}^{*}=E\left[Y_{i} \mid X_{i}=x_{i}, Y_{i}>C\right]=\mu_{y_{i}}+\sigma \frac{\phi\left(z_{i}\right)}{1-\Phi\left(z_{i}\right)}
$$


em que:

$$
\begin{aligned}
& \mu_{y_{i}}=\alpha+\beta x_{i} \\
& z_{i}=\frac{\left(C-\mu_{y_{i}}\right)}{\sigma}
\end{aligned}
$$

e $\phi(z)$ e $\Phi(z)$ são as funções densidade de probabilidade e distribuição, respectivamente, de uma distribuição normal padrão. As estimativas iniciais $\hat{\alpha}^{(0)}, \hat{\beta}^{(0)} \mathrm{e}$ $\hat{\sigma}^{(0)}$, são obtidas pelo método de mínimos quadrados, utilizando os dados como se inexistisse censura. No segundo passo, o valor esperado dos dados censurados, $\mu_{y_{i}}^{*(0)}$, é obtido utilizando (1.6) e (1.8), com a substituição dos valores dos parâmetros $\alpha, \beta$ e $\sigma$, por suas estimativas iniciais $\hat{\alpha}^{(0)}, \hat{\beta}^{(0)}$ e $\hat{\sigma}^{(0)}$. Na iteração seguinte, os valores censurados são substituídos pelos valores estimados, $\mu_{y_{i}}^{*(0)}$. Então, novas estimativas de $\alpha, \beta$ e $\sigma$, são obtidas por meio do método de mínimos quadrados. Com estas novas estimativas $\left(\hat{\alpha}^{(1)}, \hat{\beta}^{(1)}\right.$ e $\left.\hat{\sigma}^{(1)}\right)$, os valores esperados dos dados censurados são re-estimados e o processo se repete até que ocorra a convergência.

Aitkin (1981) aplicou o algoritmo EM (Dempster et al., 1977) para calcular as estimativas de máxima verossimilhança, comprovou que o método proposto por Schmee e Hahn (1979) gera estimativas diferentes daquelas obtidas por máxima verossimilhança apenas para $\sigma^{2}$ e sugeriu o uso de uma correção para amenizar o viés da estimativa de $\sigma^{2}$ obtida por máxima verossimilhança.

Chatterjee e McLeish (1986) propuseram uma modificação do método de Schmee e Hahn (1979) e explicitaram as equações de máxima verossimilhança para o caso em que a variável resposta de um modelo de regressão linear múltipla está sujeita à censura aleatória (este tipo de censura ocorre quando, o valor observado é dado por $\min \left(T_{i}, C_{i}\right)$, onde $T_{i}$ e $C_{i}$ são variáveis aleatórias independentes, que representam os valores de interesse e de censura, respectivamente, para a i-ésima unidade amostral). Sharma et al. (1995) estenderam os resultados de Schmee e Hahn (1979) para o caso em que a variável resposta está sujeita à censura intervalar.

Thompson e Nelson (2003) estenderam os resultados de Aitkin (1981) para o caso em que a variável resposta está sujeita a censura à esquerda ou à censura intervalar. Esses autores encontraram este tipo de censura ao analisar níveis de con- 
taminante classificados como quantificável, detectado mas não quantificável e não detectado. No primeiro caso o valor do nível de contaminante, $y_{i}$, da i-ésima unidade amostral é conhecido, no segundo caso, sabe-se apenas que $L D<y_{i}<L Q$ em que $L D$ e $L Q$ representam os limites de detecção e quantiicação, respectivamente $(0<L D<L Q)$ e no terceiro caso, sabemos apenas que $y_{i}<L D$. Para avaliar as estimativas obtidas por máxima verossimilhança, os autores realizaram simulações, considerando um modelo de regressão linear simples, em que a variável resposta estava sujeita a censura à esquerda e intervalar. Compararam os resultados com aqueles obtidos pela substituição dos valores censurados, pelo valor do ponto médio dos intervalos de censura. Nesses modelos, a variável resposta considerada foi $\ln$ (Concentração do contaminante); no caso de censura à esquerda os dados foram substituídos por $\ln ((0+L D) / 2)$ e no caso de censura intervalar os dados foram substituídos por $\ln ((L D+L Q) / 2)$. A distribuição normal foi adotada para o erro do modelo. $\mathrm{O}$ erro do modelo foi simulado utilizando-se a distribuição normal e a distribuição qui-quadrado com deslocamento (utilizada para verificar a robustez dos estimadores para os casos assimétricos). Quando todos os dados detectados mas não quantificáveis $\left(L D<y_{i}<L Q\right)$ foram considerados como censurados à esquerda $\left(y_{i}<L Q\right)$, o método de substituição resultou em estimativas com grau considerável de viés. As estimativas obtidas por máxima verossimilhança apresentaram pouco viés nas simulações em que o erro do modelo tinha distribuição normal, porém o método por máxima verossimilhança não se mostrou robusto para estimar $\beta$ nas simulações em que o erro não tinha distribuição normal.

Nos estudos considerados anteriormente, o objetivo principal era determinar métodos para estimar os parâmetros de um modelo de regressão linear em que o erro tem distribuição normal e no qual a variável resposta está sujeita a censura. Lachenbruch et al. (1995) estudaram o modelo de regressão linear simples, com variável independente sujeita à censura do Tipo I à direita e propuseram a substituição dos dados censurados, $X_{i}^{*}=\min \left(X_{i}, C\right)$, pelo valor esperado $E\left[X_{i} \mid X_{i}>C\right]$. Concluíram que as estimativas obtidas por este método são não enviesadas.

Austin e Brunner (2003) estudaram um modelo de regressão linear com duas 
variáveis independentes (com distribuição normal bivariada), em que uma das variáveis estava sujeita à censura do Tipo I à direita. Através de simulações, concluíram que a variável independente sujeita à censura pode aumentar a probabilidade de erro do Tipo I da outra variável independente. Sob este mesmo modelo, Austin e Hoch (2004), compararam as estimativas obtidas por cinco métodos diferentes: método de mínimos quadrados considerando todos os dados como se a censura inexistisse, método de mínimos quadrados considerando apenas os dados não censurados, método por máxima verossimilhança considerando a censura e dois métodos em que os valores censurados são substituídos por valores esperados. Os resultados das simulações mostraram um viés menor para as estimativas obtidas por máxima verossimilhança e pelo método de mínimos quadrados considerando apenas os dados não censurados.

Gomez et al. (2003) consideraram o caso de um modelo de regressão linear simples no qual a variável independente é discreta e sujeita à censura intervalar e utilizaram o método EM para estimação dos parâmetros. Simulações realizadas para comparar estimadores obtidos sob o método proposto com estimadores de mínimos quadrados utilizados após a subsituição dos valores censurados pelos valores dos respectivos pontos médios dos intervalos de censura, indicaram melhores resultados para o método proposto pelos autores.

Neste trabalho, estendemos alguns desses resultados para o caso de um modelo de regressão linear simples em que ambas as variáveis estão sujeitas à censura intervalar. Os objetivos são propor um modelo de regressão linear simples em que ambas as variáveis estão sujeitas a censura intervalar, compararmos os estimadores de mínimos quadrados obtidos sob um modelo de regressão linear simples usual em que se desconsidera a censura com aqueles obtidos por máxima verossimilhança sob o modelo proposto e propor um método de previsão para o modelo proposto.

O modelo é descrito na Seção 2. Tanto o modelo proposto quanto o modelo de regressão linear simples em que se desconsidera a censura são ajustados a dados como da Tabela 1.1 para tons de 0,5, 1,0, 2,0 e 4,0kHz de cada uma das orelhas e são apresentados na Seção 3. O método de previsão dos valores de ATL é 
apresentado na Seção 4. Na Seção 5, consideramos um estudo de simulação para comparar os estimadores de máxima verossimilhança sob o modelo proposto com os estimadores do modelo de regressão linear simples usual em que se desconsidera a censura e a discussão dos resultados é feita na Seção 6. 



\section{Capítulo 2}

\section{Modelo e função de verossimilhança}

Para incorporar a censura das duas variáveis ao modelo de regressão linear simples (1.1), vamos assumir que a variável aleatória $X_{i}$ tem distribuição normal e que $X_{i}$ e $\epsilon_{i}$ são independentes. $O$ modelo resultante é:

$$
Y_{i}=\alpha+\beta X_{i}+\epsilon_{i}
$$

em que $Y_{i}$ é uma variável aleatória que representa o valor de $Y$ da i-ésima unidade amostral, $X_{i}$ é uma variável aleatória que representa o valor de $X$ da i-ésima unidade amostral, consideramos que $X_{i}$ são variáveis aleatórias independentes, cuja distribuição é $N\left(\mu_{x}, \sigma_{x}^{2}\right), \epsilon_{i}$ são variáveis aleatórias independentes, cuja distribuição é $N\left(0, \sigma^{2}\right), X_{i}$ e $\epsilon_{i}$ são independentes, $i=1 \ldots n$, em que $n$ é o tamanho da amostra. Os parâmetros do modelo são agrupados no vetor $\boldsymbol{\theta}=\left(\alpha, \beta, \sigma, \mu_{x}, \sigma_{x}\right)$.

Como todos os valores de $Y_{i}$ e $X_{i}$ são censurados de forma intervalar, a resposta do i-ésimo indivíduo pode ser representada pelo vetor de variáveis aleatórias $\left(Y_{\text {infi }}, Y_{\text {sup } i}, X_{\text {inf }}, X_{\text {sup }}\right)$, que são os limites inferior e superior dos intervalos de censura de $Y_{i}$ e de $X_{i}$, respectivamente. Assumimos que $Y_{\text {inf }}<Y_{\text {sup }_{i}}$ e que $X_{\text {inf }_{i}}<X_{\text {sup }_{i}}, i=1 \ldots n$. A função de densidade de probabilidade de $Y_{i}$ condicionada aos valores de $X_{i}=x$ e a função densidade de probabilidade de $X_{i}$ são dadas, 
respectivamente, por

$$
\begin{gathered}
f(y \mid x, \alpha, \beta, \sigma)=\frac{1}{\sqrt{2 \pi \sigma^{2}}} \exp \left(-\frac{(y-(\alpha+\beta x))^{2}}{2 \sigma^{2}}\right) \\
f\left(x \mid \mu_{x}, \sigma_{x}\right)=\frac{1}{\sqrt{2 \pi \sigma_{x}^{2}}} \exp \left(-\frac{\left(x-\mu_{x}\right)^{2}}{2 \sigma_{x}^{2}}\right)
\end{gathered}
$$

A função de verossimilhança $L\left(\boldsymbol{\theta} ; \mathbf{y}_{\text {inf }}, \mathbf{y}_{\text {sup }}, \mathbf{x}_{\text {inf }}, \mathbf{x}_{\text {sup }}\right)=L(\boldsymbol{\theta})$ é dada por

$$
L(\boldsymbol{\theta})=\prod_{i=1}^{n} \int_{y_{i n f_{i}}}^{y_{\text {sup }_{i}}} \int_{x_{i n f_{i}}}^{x_{\text {sup }_{i}}} f(y \mid x, \alpha, \beta, \sigma) f\left(x \mid \mu_{x}, \sigma_{x}\right) d x d y
$$

em que $\mathbf{y}_{\text {inf }}=\left(y_{\text {inf }}, y_{\text {inf }}, \ldots, y_{\text {inf }}\right), \quad \mathbf{y}_{\text {sup }}=\left(y_{\text {sup } 1}, y_{\text {sup } 2}, \ldots, y_{\text {sup }}\right)$, $\mathbf{x}_{\text {inf }}=\left(x_{i n f_{1}}, x_{i n f_{2}}, \ldots, x_{i n f_{n}}\right)$ e $\mathbf{x}_{\text {sup }}=\left(x_{\text {sup }}, x_{\text {sup }}, \ldots, x_{\text {sup }}\right)$, são os valores observados de $Y_{\text {inf }}, Y_{\text {sup }_{i}}, X_{\text {inf }}$ e $X_{\text {sup }_{i}}$, para $i=1, . ., n$, respectivamente.

As estimativas de máxima verossimilhança $\left(\hat{\boldsymbol{\theta}}_{M V}\right)$ podem ser calculadas por meio do método iterativo de Newton-Raphson, veja, por exemplo, Thisted (1988), aplicado ao logaritmo da função de verossimilhança, $l(\boldsymbol{\theta})=\ln (L(\boldsymbol{\theta}))$ e é dado por

$$
l(\boldsymbol{\theta})=\sum_{i=1}^{n} \ln \left(\int_{y_{\text {inf }}}^{y_{\text {sup }}} \int_{x_{i n f_{i}}}^{x_{\text {sup }_{i}}} f(y \mid x, \alpha, \beta, \sigma) f\left(x \mid \mu_{x}, \sigma_{x}\right) d x d y\right)
$$

O cálculo da estimativa $\left(\hat{\boldsymbol{\theta}}_{M V}\right)$ através do método de Newton-Raphson, as expressões das derivadas parciais, $\dot{\boldsymbol{l}}(\boldsymbol{\theta})$, e da matrix Hessiana, $\ddot{\boldsymbol{l}}(\boldsymbol{\theta})$, são apresentadas nos Apêndices A e B, respectivamente. Utilizamos o pacote computacional Maple 9.5 (Maplesoft, 2004) para realizá-los. 


\section{Capítulo 3}

\section{Ajuste dos modelos}

O ajuste do modelo de regressão linear simples usual foi realizado considerandose valores de ATL e RAEE utilizados na clínica como limiares. Exemplos destes valores podem ser vistos na Tabela 1.1. Denotamos os valores obtidos por ATL e por RAEE, para o i-ésimo indivíduo por $Y_{i}$ e $X_{i}$, respectivamente.

Para o modelo proposto denotamos $Y_{\text {inf }}$ e $Y_{\text {sup }}$, respectivamente, os limites inferior e superior de censura do limiar de ATL do i-ésimo indivíduo, e $X_{i n f_{i}}$ e $X_{\text {sup }_{i}}$, respectivamente, como os limites inferior e superior de censura do limiar de RAEE do i-ésimo indivíduo. Exemplos de intervalos de censura observados sob os métodos ATL e RAEE podem ser vistos na Tabela 1.1.

Os resultados do ajuste dos dois modelos para cada tom e orelha encontramse na Tabela 3.1. Em geral, se compararmos o modelo proposto com o usual, notamos um aumento nos valores das estimativas de $\alpha(\hat{\alpha})$ e uma diminuição das estimativas de $\beta(\hat{\beta})$.

Apesar disso, observando as Figuras 3.1 e 3.2, não é possível perceber diferenças relevantes entre os resultados obtidos sob os dois modelos. A maior diferença encontrada, foi para o tom de freqüência de $4,0 \mathrm{kHz}$ na orelha esquerda: aumento na estimativa de $\alpha$ de $47 \%$ e diminuição na estimativa de $\beta$ de $10 \%$. Com relação as estimativas de $\sigma(\hat{\sigma})$, as diferenças absolutas encontradas foram no máximo de 1 dBNA.

Definimos o resíduo ordinário do ponto médio $\left(e_{i}^{*}\right)$ para o i-ésimo indivíduo 
Tabela 3.1: Estimativas dos parâmetros do modelo de regressão usual e do modelo de regressão proposto e a variação percentual dos valores obtidos sob o modelo proposto em relação ao modelo usual

\begin{tabular}{|c|c|c|c|c|c|c|c|c|c|c|}
\hline \multirow{2}{*}{ Freqüência (kHz) } & \multirow{2}{*}{ Orelha } & \multicolumn{3}{|c|}{ Modelo usual } & \multicolumn{3}{|c|}{ Modelo proposto } & \multicolumn{3}{|c|}{ Variação \% } \\
\hline & & $\hat{\alpha}(\mathrm{EP})$ & $\hat{\beta}(\mathrm{EP})$ & $\hat{\sigma}$ & $\hat{\alpha}(\mathrm{EP})$ & $\hat{\beta}(\mathrm{EP})$ & $\hat{\sigma}$ & $\hat{\alpha}$ & $\hat{\beta}$ & $\hat{\sigma}$ \\
\hline 0 & OD & $-19(6)$ & $1,03(0,07)$ & 13 & $-15(6)$ & $0,97(0,07)$ & 13 & 18 & -6 & 0 \\
\hline & $\mathrm{OE}$ & $-13(7)$ & $0,97(0,08)$ & 15 & $-11(7)$ & $0,93(0,08)$ & 15 & 15 & -3 & 1 \\
\hline \multirow{2}{*}{1,0} & OD & $-10(5)$ & $1,00(0,06)$ & 11 & $-9(5)$ & $0,98(0,06)$ & 11 & 9 & -2 & -1 \\
\hline & $\mathrm{OE}$ & $-9(6)$ & $0,99(0,07)$ & 14 & $-11(6)$ & $1,02(0,08)$ & 15 & -17 & 3 & 5 \\
\hline \multirow{2}{*}{2,0} & OD & $-6(4)$ & $0,97(0,05)$ & 11 & $-5(4)$ & $0,95(0,06)$ & 11 & 20 & -2 & 0 \\
\hline & $\mathrm{OE}$ & $-7(5)$ & $1,00(0,06)$ & 11 & $-7(5)$ & $1,00(0,07)$ & 12 & -1 & 0 & 3 \\
\hline \multirow{2}{*}{4,0} & OD & $-13(6)$ & $1,01(0,07)$ & 14 & $-13(7)$ & $0,98(0,08)$ & 15 & 5 & -2 & 8 \\
\hline & $\mathrm{OE}$ & $-11(6)$ & $0,97(0,07)$ & 13 & $-6(6)$ & $0,87(0,07)$ & 13 & 47 & -10 & -2 \\
\hline
\end{tabular}


como

$$
e_{i}^{*}=y_{p m, i}-\hat{y}_{p m, i}, i \in C
$$

em que

$$
\hat{y}_{p m, i}=\hat{\alpha}_{M V}+\hat{\beta}_{M V} x_{p m, i}, i \in C,
$$

$y_{p m, i}$ é o ponto médio do intervalo $\left(y_{i n f_{i}}, y_{\text {sup }_{i}}\right], x_{p m, i}$ é o ponto médio do intervalo $\left(x_{i n f_{i}}, x_{\text {sup }}\right], \hat{\alpha}_{M V}$ e $\hat{\beta}_{M V}$ são as estimativas de máxima verossimilhança de $\alpha$ e $\beta$ sob o modelo proposto e $C=\left\{i \mid-\infty<x_{\text {inf }}, y_{\text {inf }}, x_{\text {sup }_{i}}, y_{\text {sup }_{i}}<\infty\right\}$.

Para avaliar se a suposição de homocedasticidade do modelo proposto é aceitável, gráficos de dispersão entre os valores dos resíduos do ponto médio padronizados $\left(e_{i}^{*} / \sigma_{M V}\right)$ e os valores ajustados do ponto médio $\left(\hat{y}_{p m, i}\right)$ estão apresentados nas Figuras 3.3 e 3.4. Nenhum deles sugere heterocedasticidade.

Para avaliar a suposição de normalidade de $\epsilon_{i}$ no modelo proposto, os limites inferior e superior de censura dos resíduos foram calculados. Para isto, consideramos

$$
\hat{y}(x)=\hat{\alpha}_{M V}+\hat{\beta}_{M V} x
$$

e definimos

$$
\begin{aligned}
\operatorname{lr} p_{\text {inf }} & =\frac{1}{\hat{\sigma}_{M V}} \min \left(f 1_{i}, f 2_{i}, f 3_{i}, f 4_{i}\right) \\
\operatorname{lr} p_{\text {sup }_{i}} & =\frac{1}{\hat{\sigma}_{M V}} \max \left(f 1_{i}, f 2_{i}, f 3_{i}, f 4_{i}\right)
\end{aligned}
$$

com

$$
\begin{aligned}
f 1_{i} & =\lim _{\substack{y \rightarrow y_{\text {in }} \\
x \rightarrow x_{\text {inf }}}}(y-\hat{y}(x)) \\
f 2_{i} & =\lim _{\substack{y \rightarrow y_{\text {sup }} \\
x \rightarrow x_{\text {inf }}}}(y-\hat{y}(x)) \\
f 3_{i} & =\lim _{\substack{y \rightarrow y_{\text {in }} \\
x \rightarrow \text { sup }_{i}}}(y-\hat{y}(x)) \\
f 4_{i} & =\lim _{\substack{y \rightarrow y_{\text {sup }} \\
x \rightarrow x_{\text {sup }}}}(y-\hat{y}(x)) .
\end{aligned}
$$


Figura 3.1: Ajustes do modelo usual e do modelo proposto para as frequiências de 0,5 e $1,0 \mathrm{kHz}$.

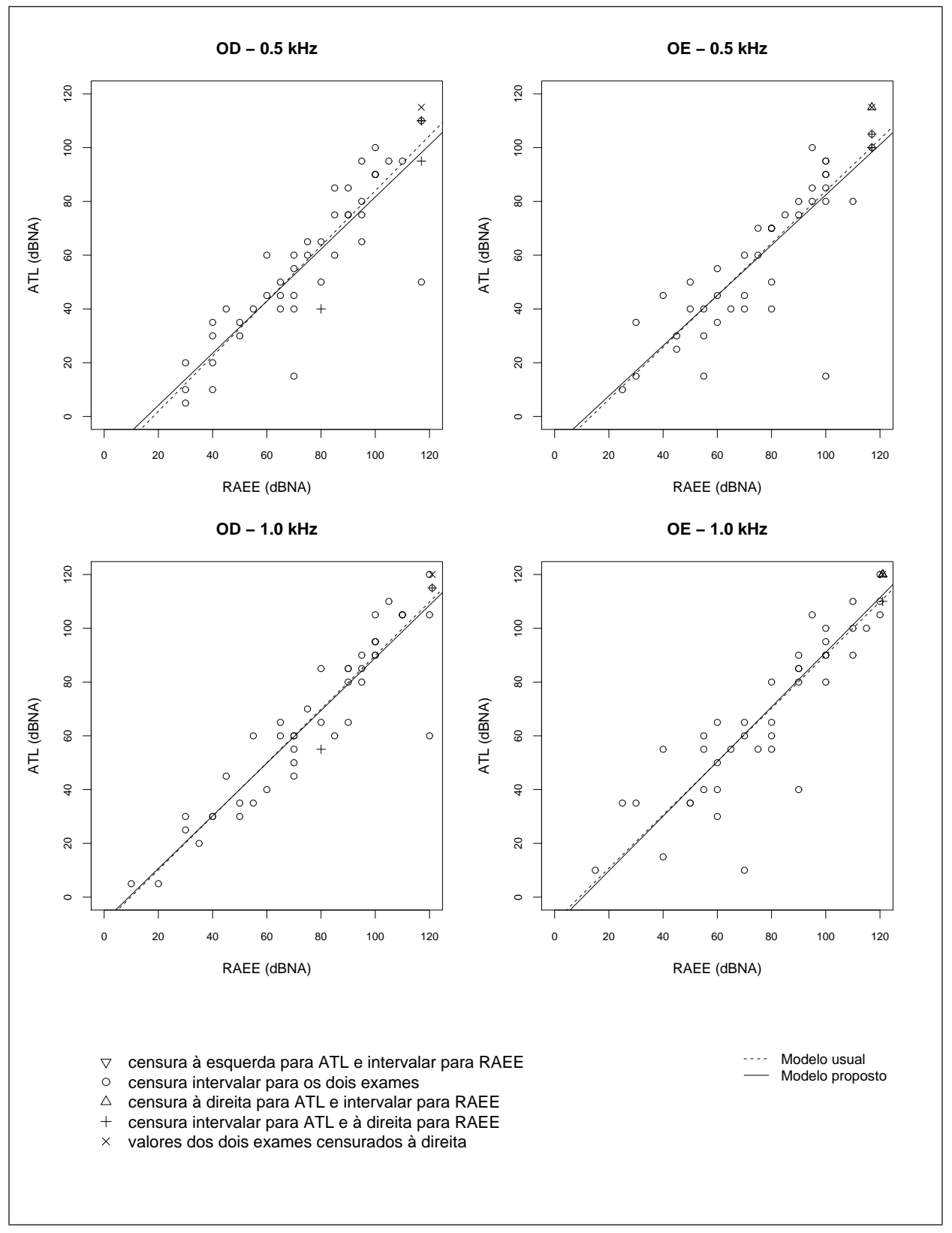


Figura 3.2: Ajustes do modelo usual e do modelo proposto para as frequiências de 2,0 e $4,0 \mathrm{kHz}$.

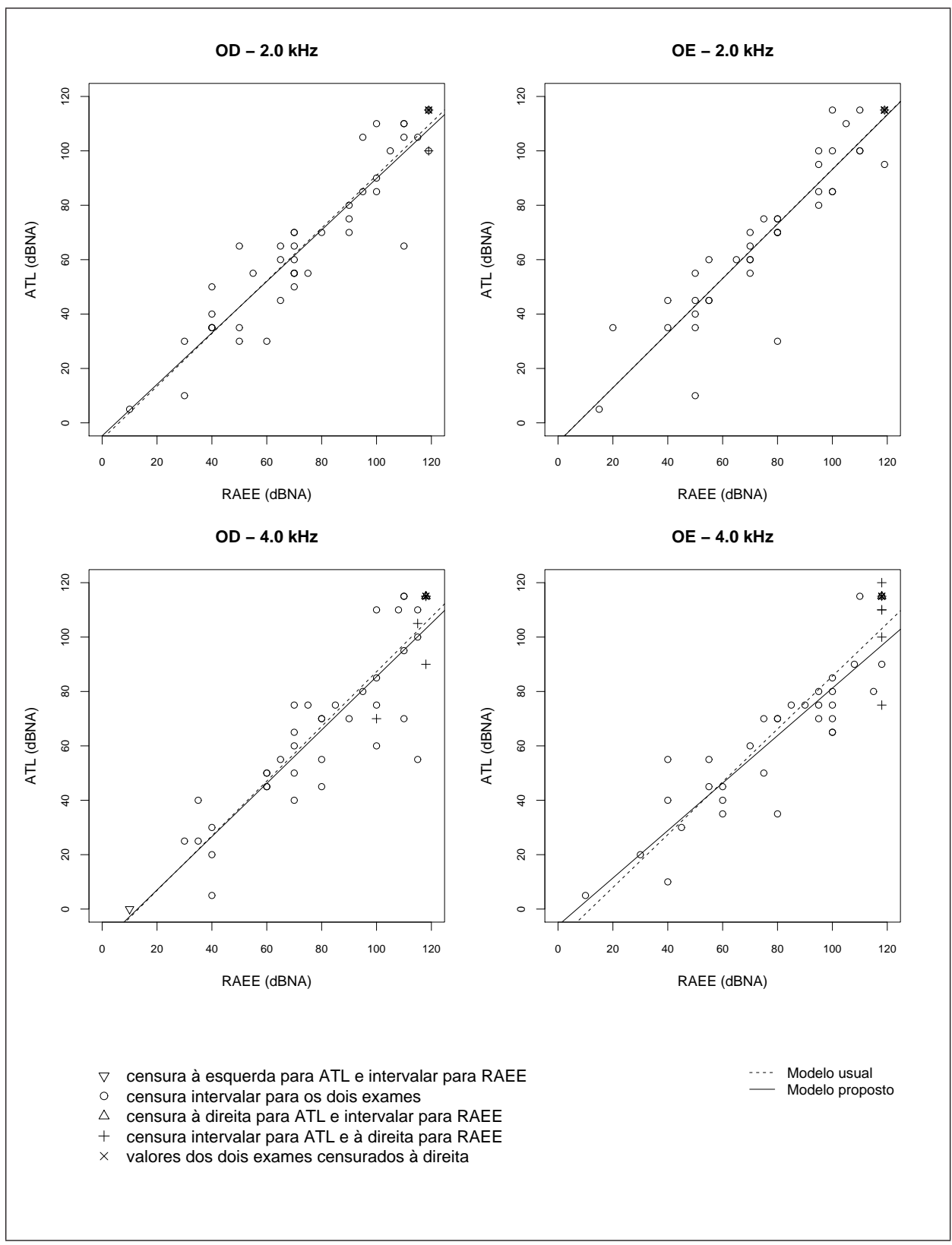


Figura 3.3: Gráficos de dispersão entre os resíduos do ponto médio padronizados $\left(e_{i}^{*} / \sigma_{M V}\right)$ e os valores ajustados do ponto médio $\left(\hat{y}_{p m, i}\right)$ para o modelo proposto para cada orelha para as freqüências de 0,5 e $1,0 \mathrm{kHz}$

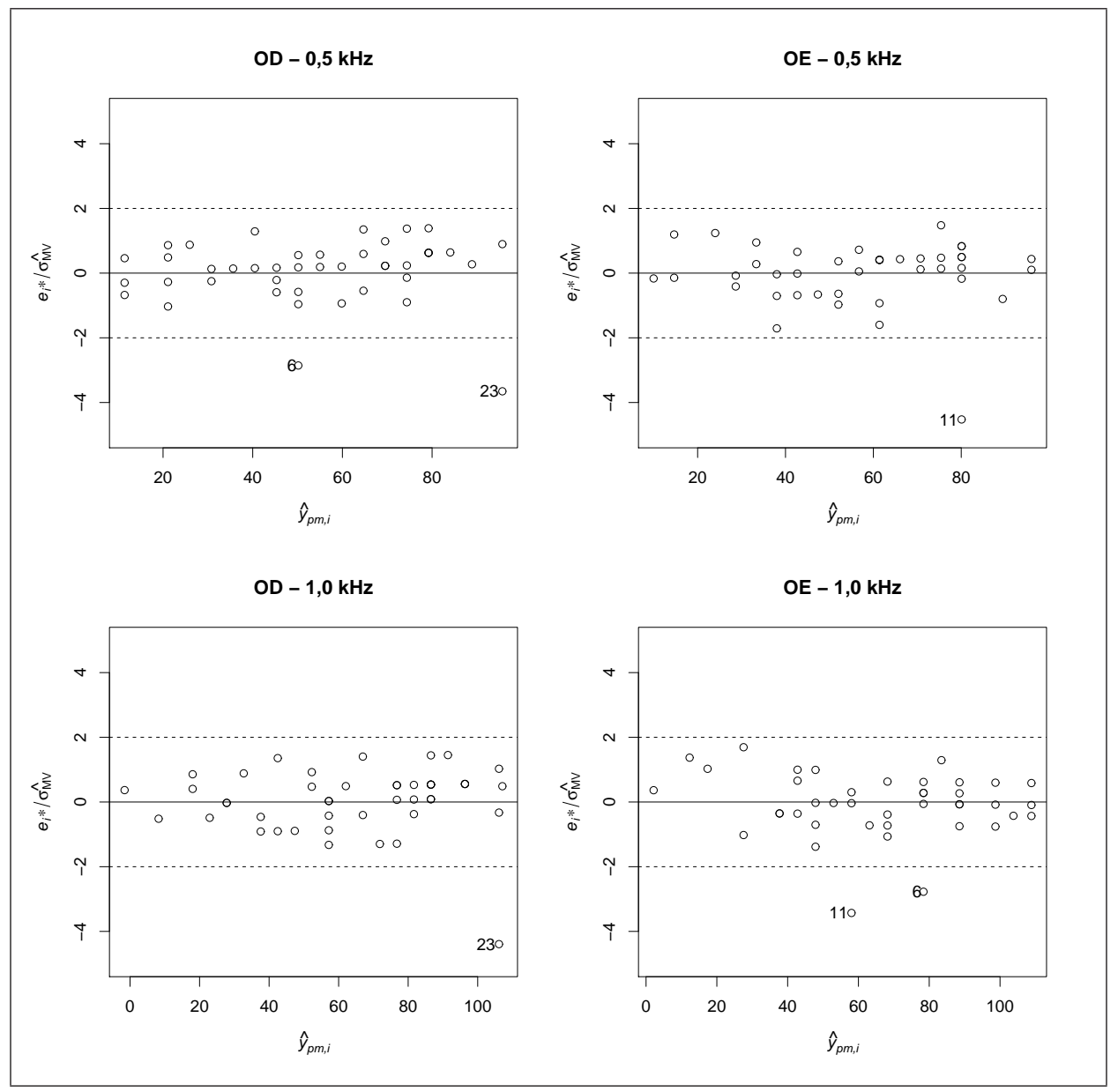


Figura 3.4: Gráficos de dispersão entre os resíduos do ponto médio padroniza$\operatorname{dos}\left(e_{i}^{*} / \sigma_{M V}\right)$ e os valores ajustados do ponto médio $\left(\hat{y}_{p m, i}\right)$ para o modelo proposto para cada orelha para as freqüências de 2,0 e $4,0 \mathrm{kHz}$

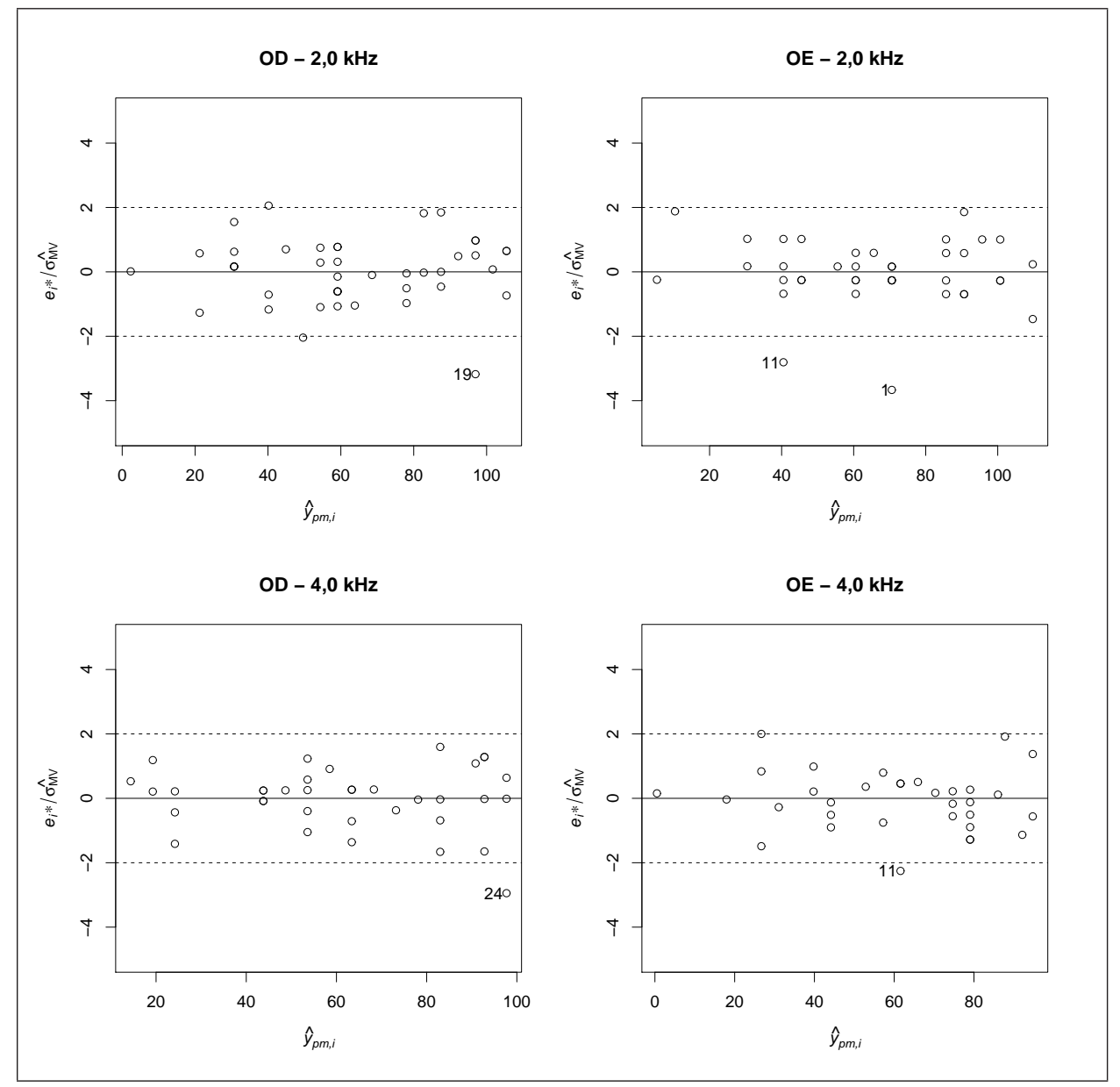


Figura 3.5: Exemplo do cálculo de $f 1_{i}, f 2_{i}, f 3_{i}$ e $f 4_{i}$, considerando os valores: $\left(x_{\text {inf }}, x_{\text {sup }_{i}}\right]=(60,65]$ e $\left(y_{\text {inf }}, y_{\text {sup }_{i}}\right]=(70,75]$

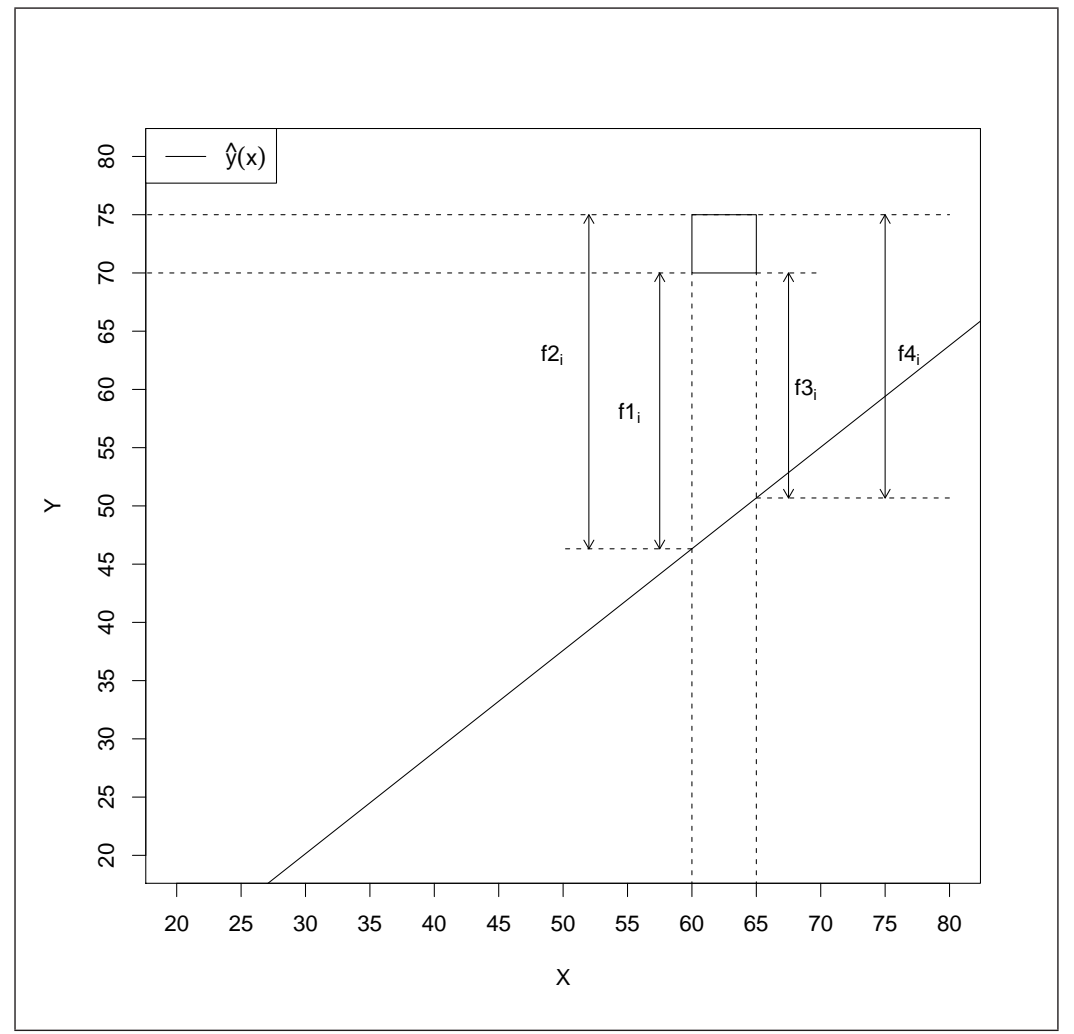

Os termos $\operatorname{lr} p_{\text {inf }}$ e $\operatorname{lr} p_{\text {sup }}$, correspondem respectivamente, aos limites inferior e superior de censura do i-ésimo resíduo padronizado, em que $\hat{\sigma}_{M V}$ é a estimativa de máxima verossimilhança de $\sigma$ sob o modelo proposto.

Na Figura 3.5 apresentamos um exemplo do cálculo de $f 1_{i}, f 2_{i}, f 3_{i}$ e $f 4_{i}$ considerando os intervalos: $\left(x_{\text {inf }}, x_{\text {sup }_{i}}\right]=(60,65]$ e $\left(y_{\text {inf }}, y_{\text {sup }_{i}}\right]=(70,75]$. Na Tabela 3.2, encontram-se os limites de censura do modelo ajustado para os dados da orelha direita para o tom de 4,0 kHz. Podemos observar, que para os pares em que ATL e RAEE são censurados à direita, não é possível definir os limites de censura dos resíduos. 
Tabela 3.2: Intervalos de censura do limiar auditivo e eletrofisiológico (dBNA), obtidos por ATL e RAEE da orelha direita (OD) para um tom com freqüência de $4,0 \mathrm{kHz}$ e valores de $f 1_{i}, f 2_{i}, f 3_{i}$ e $f 4_{i}$ e dos limites de censura dos resíduos padronizados

\begin{tabular}{cccccccc}
\hline Indivíduo & ATL & RAEE & $f 1_{i}$ & $f 2_{i}$ & $f 3_{i}$ & $f 4 i$ & $\begin{array}{c}\text { Resíduo } \\
\text { padronizado }\end{array}$ \\
\hline 1 & & & & & & & \\
2 & $(70 ; 75]$ & $(70 ; 75]$ & 14,0 & 19,0 & 9,1 & 14,1 & $(0,59 ; 1,24]$ \\
3 & $(0 ; 5]$ & $(35 ; 40]$ & $-21,7$ & $-16,7$ & $-26,6$ & $-21,6$ & $(-1,74 ;-1,09]$ \\
4 & $(15 ; 20]$ & $(35 ; 40]$ & $-6,7$ & $-1,7$ & $-11,6$ & $-6,6$ & $(-0,76 ;-0,11]$ \\
5 & $(35 ; 40]$ & $(30 ; 35]$ & 18,2 & 23,2 & 13,3 & 18,3 & $(0,86 ; 1,51]$ \\
6 & $(35 ; 40]$ & $(65 ; 70]$ & $-16,1$ & $-11,1$ & $-21,0$ & $-16,0$ & $(-1,37 ;-0,73]$ \\
7 & $(50 ; 55]$ & $(75 ; 80]$ & $-10,9$ & $-5,9$ & $-15,8$ & $-10,8$ & $(-1,03 ;-0,39]$ \\
8 & $(25 ; 30]$ & $(35 ; 40]$ & 3,3 & 8,3 & $-1,6$ & 3,4 & $(-0,11 ; 0,54]$ \\
9 & $(40 ; 45]$ & $(75 ; 80]$ & $-20,9$ & $-15,9$ & $-25,8$ & $-20,8$ & $(-1,68 ;-1,04]$ \\
10 & $(20 ; 25]$ & $(30 ; 35]$ & 3,2 & 8,2 & $-1,7$ & 3,3 & $(-0,11 ; 0,53]$ \\
11 & $(65 ; 70]$ & $(75 ; 80]$ & 4,1 & 9,1 & $-0,8$ & 4,2 & $(-0,06 ; 0,59]$ \\
12 & $(55 ; 60]$ & $(65 ; 70]$ & 3,9 & 8,9 & $-1,0$ & 4,0 & $(-0,07 ; 0,58]$ \\
13 & $(45 ; 50]$ & $(55 ; 60]$ & 3,7 & 8,7 & $-1,2$ & 3,8 & $(-0,08 ; 0,56]$ \\
14 & $(45 ; 50]$ & $(55 ; 60]$ & 3,7 & 8,7 & $-1,2$ & 3,8 & $(-0,08 ; 0,56]$ \\
15 & $(60 ; 65]$ & $(65 ; 70]$ & 8,9 & 13,9 & 4,0 & 9,0 & $(0,26 ; 0,90]$ \\
16 & $(40 ; 45]$ & $(55 ; 60]$ & $-1,3$ & 3,7 & $-6,2$ & $-1,2$ & $(-0,41 ; 0,24]$ \\
17 & $(55 ; 60]$ & $(95 ; 100]$ & $-25,5$ & $-20,5$ & $-30,5$ & $-25,5$ & $(-1,98 ;-1,34]$ \\
18 & $(70 ; 75]$ & $(80 ; 85]$ & 4,2 & 9,2 & $-0,7$ & 4,3 & $(-0,05 ; 0,60]$ \\
19 & $(115 ;+\infty]$ & $(113 ; 118]$ & 16,8 & $+\infty$ & 11,9 & $+\infty$ & $(0,78 ;+\infty)$ \\
20 & $(65 ; 70]$ & $(105 ; 110]$ & $-25,4$ & $-20,4$ & $-30,3$ & $-25,3$ & $(-1,97 ;-1,33]$ \\
21 & $(45 ; 50]$ & $(65 ; 70]$ & $-6,1$ & $-1,1$ & $-11,0$ & $-6,0$ & $(-0,72 ;-0,07]$ \\
22 & $(50 ; 55]$ & $(60 ; 65]$ & 3,8 & 8,8 & $-1,1$ & 3,9 & $(-0,07 ; 0,57]$ \\
23 & $(65 ; 70]$ & $(100 ;+\infty]$ & $-20,5$ & $-15,5$ & $-\infty$ & $-\infty$ & $(-\infty ;-1,01]$ \\
24 & $(65 ; 70]$ & $(75 ; 80]$ & 4,1 & 9,1 & $-0,8$ & 4,2 & $(-0,06 ; 0,59]$ \\
\hline & & & & & & & continua
\end{tabular}




\begin{tabular}{|c|c|c|c|c|c|c|c|}
\hline Indivíduo & ATL & RAEE & $f 1_{i}$ & $f 2_{i}$ & $f 3_{i}$ & $f 4_{i}$ & $\begin{array}{c}\text { Resíduo } \\
\text { padronizado }\end{array}$ \\
\hline 25 & $(40 ; 45]$ & $(55 ; 60]$ & $-1,3$ & 3,7 & $-6,2$ & $-1,2$ & $(-0,41 ; 0,24]$ \\
\hline 26 & $(50 ; 55]$ & $(110 ; 115]$ & $-45,3$ & $-40,3$ & $-50,2$ & $-45,2$ & $(-3,27 ;-2,62]$ \\
\hline 27 & $(80 ; 85]$ & $(95 ; 100]$ & $-0,5$ & 4,5 & $-5,5$ & $-0,5$ & $(-0,36 ; 0,29]$ \\
\hline 28 & $(65 ; 70]$ & $(85 ; 90]$ & $-5,7$ & $-0,7$ & $-10,6$ & $-5,6$ & $(-0,69 ;-0,05]$ \\
\hline 29 & $(100 ; 105]$ & $(115 ;+\infty]$ & $-0,2$ & 4,8 & $-\infty$ & $-\infty$ & $(-\infty ; 0,32]$ \\
\hline 30 & $(115 ;+\infty]$ & $(118 ;+\infty]$ & 11,9 & $+\infty$ & $-\infty$ & & \\
\hline 31 & $(95 ; 100]$ & $(110 ; 115]$ & $-0,3$ & 4,7 & $-5,2$ & $-0,2$ & $(-0,34 ; 0,31]$ \\
\hline 32 & $(70 ; 75]$ & $(65 ; 70]$ & 18,9 & 23,9 & 14,0 & 19,0 & $(0,91 ; 1,55]$ \\
\hline 33 & $(70 ; 75]$ & $(95 ; 100]$ & $-10,5$ & $-5,5$ & $-15,5$ & $-10,5$ & $(-1,01 ;-0,36]$ \\
\hline 34 & $(90 ; 95]$ & $(105 ; 110]$ & $-0,4$ & 4,6 & $-5,3$ & $-0,3$ & $(-0,34 ; 0,30]$ \\
\hline 35 & $(75 ; 80]$ & $(90 ; 95]$ & $-0,6$ & 4,4 & $-5,5$ & $-0,5$ & $(-0,36 ; 0,28]$ \\
\hline 36 & $(110 ; 115]$ & $(105 ; 110]$ & 19,6 & 24,6 & 14,7 & 19,7 & $(0,96 ; 1,61]$ \\
\hline 37 & $(85 ; 90]$ & $(118 ;+\infty]$ & $-18,1$ & $-13,1$ & $-\infty$ & $-\infty$ & $(-\infty ;-0,85]$ \\
\hline 38 & $(110 ; 115]$ & $(118 ;+\infty]$ & 6,9 & 11,9 & $-\infty$ & $-\infty$ & $(-\infty ; 0,78]$ \\
\hline 39 & $(105 ; 110]$ & $(103 ; 108]$ & 16,6 & 21,6 & 11,7 & 16,7 & $(0,76 ; 1,41]$ \\
\hline 40 & $(105 ; 110]$ & $(95 ; 100]$ & 24,5 & 29,5 & 19,5 & 24,5 & $(1,27 ; 1,92]$ \\
\hline 41 & $(-\infty ; 0]$ & $(5 ; 10]$ & $-\infty$ & 7,7 & $-\infty$ & 2,8 & $(-\infty ; 0,50]$ \\
\hline 42 & $(115 ;+\infty]$ & $(118 ;+\infty]$ & 11,9 & $+\infty$ & $-\infty$ & & \\
\hline 43 & $(110 ; 115]$ & $(105 ; 110]$ & 19,6 & 24,6 & 14,7 & 19,7 & $(0,96 ; 1,61]$ \\
\hline 44 & $(110 ; 115]$ & $(118 ;+\infty]$ & 6,9 & 11,9 & $-\infty$ & $-\infty$ & $(-\infty ; 0,78]$ \\
\hline 45 & $(105 ; 110]$ & $(110 ; 115]$ & 9,7 & 14,7 & 4,8 & 9,8 & $(0,32 ; 0,96]$ \\
\hline 46 & $(115 ;+\infty]$ & $(118 ;+\infty]$ & 11,9 & $+\infty$ & $-\infty$ & & \\
\hline 47 & $(115 ;+\infty]$ & $(113 ; 118]$ & 16,8 & $+\infty$ & 11,9 & $+\infty$ & $(0,78 ;+\infty)$ \\
\hline 48 & $(115 ;+\infty]$ & $(118 ;+\infty]$ & 11,9 & $+\infty$ & $-\infty$ & & \\
\hline
\end{tabular}

Com a informação dos intervalos de censura dos resíduos padronizados, utilizamos o método proposto por Turnbull (1976) para calcular a função de distribuição empírica $(\hat{F})$ dos mesmos. Resumidamente, seja $F(w)$ a distribuição da variável aleatória $W$ e considere valores $q_{j}$ e $p_{j}$ de $W$, tal que:

$q_{1} \leq p_{1}<q_{2} \leq \ldots<q_{m} \leq p_{m}$ 
$s_{j}=F\left(p_{j}^{+}\right)-F\left(q_{j}^{-}\right)$

$\sum_{j=1}^{m} s_{j}=1$

$s_{j} \geq 0(1 \leq j \leq m)$

$\hat{s}_{j}$ é o estimador de $s_{j}$

A distribuição empírica de $\hat{F}(w)$ de $F(w)$ é dada por:

$$
\hat{F}(w)= \begin{cases}0 & \text { se } w<q_{1}, \\ \hat{s}_{1}+\hat{s}_{2}+\ldots+\hat{s}_{j} & \text { se } p_{j}<w<q_{j+1}(1 \leq j \leq m-1) \\ 1 & \text { se } w>p_{m},\end{cases}
$$

e indefinida para $w \in\left[q_{j}, p_{j}\right]$ para $1 \leq j \leq m$. Considerando os quantis de $\hat{F}(w)$, dados por $\hat{F}\left(q_{j+1}^{-}\right)=\hat{s}_{1}+\ldots+\hat{s}_{j}(1 \leq j \leq m-1)$, construímos os gráficos de dispersão entre estes quantis amostrais e os quantis teóricos considerando uma distribuição normal. Os cálculos foram realizados no pacote computacional SAS 9.1 (SAS Institute Inc, 2004). Os gráficos de dispersão entre os quantis podem ser vistos nas Figuras 3.6 e 3.7. Apesar da existência de pontos discrepantes em alguns casos (OD $0,5 \mathrm{kHz}$ e OE 2,0kHz), os gráficos sugerem que a distribuição normal pode ser utilizada como uma boa aproximação da distribuição dos resíduos do modelo.

O mesmo método foi aplicado para investigar a suposição de normalidade da variável $X_{i}$ do modelo proposto. Para calcular os quantis amostrais, foram utilizados os limites de censura dos valores de RAEE padronizados: $\left(X_{i}-\hat{\mu}_{x}\right) / \hat{\sigma}_{x}$, em que $\hat{\mu}_{x}$ e $\hat{\sigma}_{x}$ são os estimadores de máxima verossimilhança de $\mu_{x}$ e $\sigma_{x}$ sob o modelo proposto. Os gráficos podem ser vistos nas Figuras 3.8 e 3.9 e indicam que a suposição de distribuição normal de $X$ é aceitável. 
Figura 3.6: Gráficos de dispersão entre os quantis amostrais e os quantis teóricos dos resíduos padronizados para as freqüências de 0,5 e $1,0 \mathrm{kHz}$

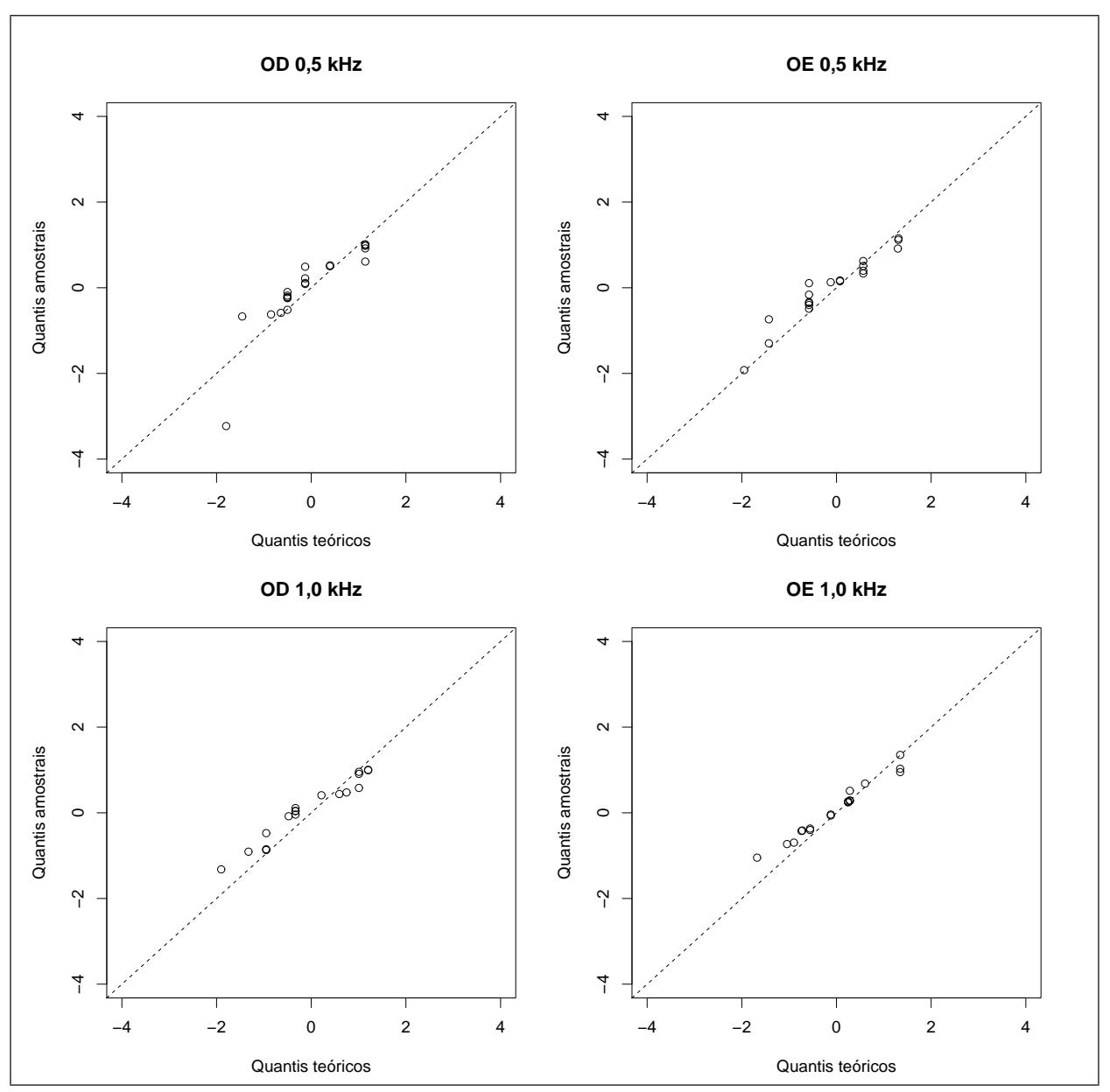


Figura 3.7: Gráficos de dispersão entre os quantis amostrais e os quantis teóricos dos resíduos padronizados para as frequiências de 2,0 e 4,0 kHz

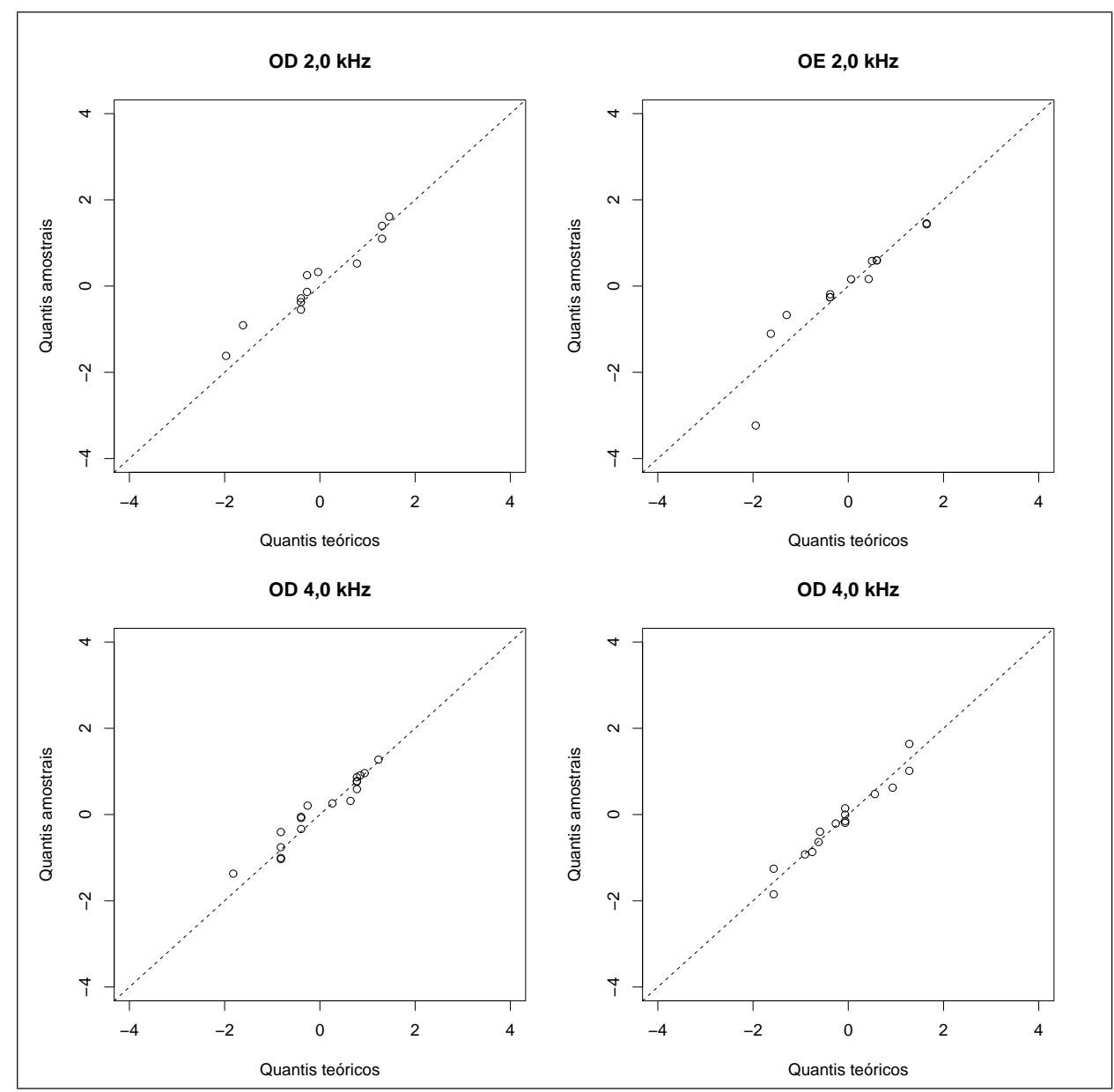


Figura 3.8: Gráficos de dispersão entre os quantis amostrais e os quantis teóricos dos valores de RAEE padronizados para as frequiências de 0,5 e $1,0 \mathrm{kHz}$

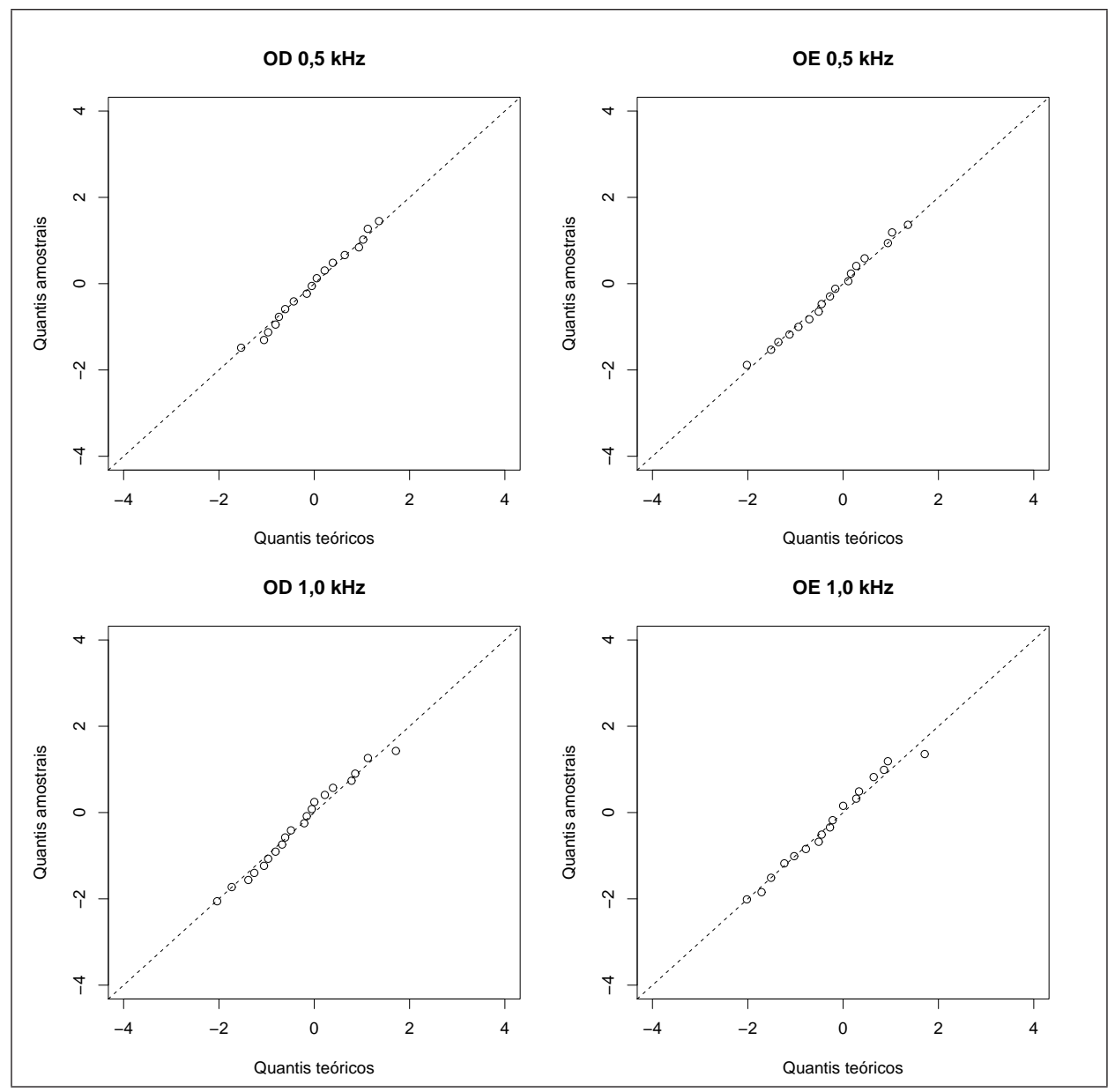


Figura 3.9: Gráficos de dispersão entre os quantis amostrais e os quantis teóricos dos valores de RAEE padronizados para as freqüências de 2,0 e 4,0 kHz

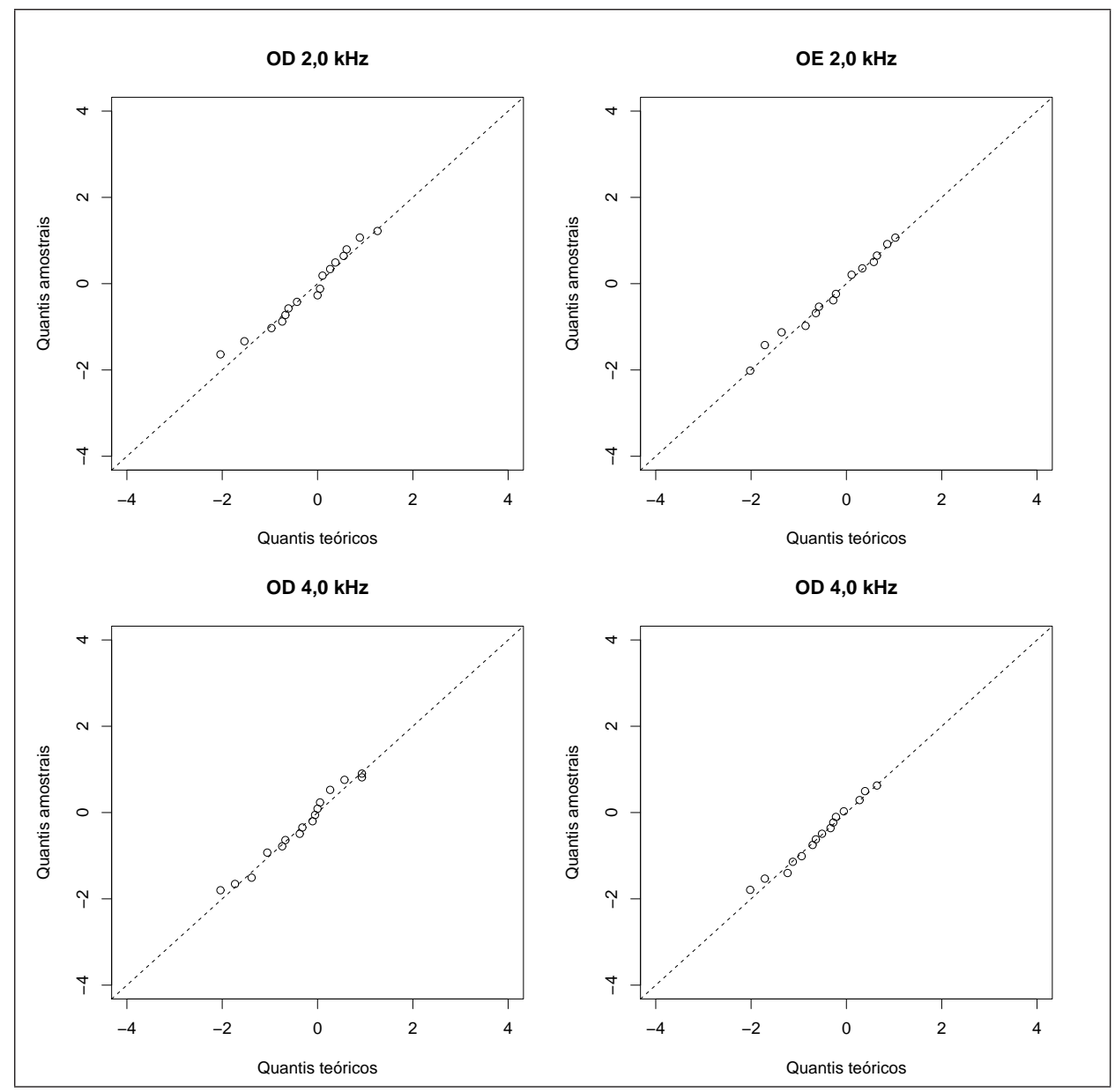





\section{Capítulo 4}

\section{Intervalos de previsão}

Seja $Y_{h}$ uma nova informação da variável aleatória $Y \mid X=x_{h}$ independente das demais. Nestas condições o intervalo de previsão de $95 \%$ para $Y_{h}$ é dado por (Casella e Berger, 1990):

$$
\boldsymbol{x}_{\boldsymbol{h}}^{t} \hat{\boldsymbol{\theta}} \pm t_{n-2,0,975}\left(\widehat{\operatorname{Var}}\left(Y_{h}-\boldsymbol{x}_{\boldsymbol{h}}^{t} \hat{\boldsymbol{\theta}}\right)\right)^{1 / 2}
$$

em que, $\boldsymbol{x}_{\boldsymbol{h}}^{t}=\left(\begin{array}{ll}1 & x_{h}\end{array}\right), \hat{\boldsymbol{\theta}}^{t}=\left(\hat{\alpha}_{M Q} \hat{\beta}_{M Q}\right)$ o vetor dos estimadores de mínimos quadrados de $\alpha, \beta$, respectivamente, $t_{(n-2,0,975)}$ é o percentil 97,5 de uma distribuição t de Student com (n-2) graus de liberdade, e $\widehat{\operatorname{Var}}\left(Y_{h}-\boldsymbol{x}_{\boldsymbol{h}}^{t} \hat{\boldsymbol{\theta}}\right)$ é o estimador de $\operatorname{Var}\left(Y_{h}-\boldsymbol{x}_{\boldsymbol{h}}^{t} \hat{\boldsymbol{\theta}}\right)$, dado por

$$
\begin{aligned}
\widehat{\operatorname{Var}}\left(Y_{h}-\boldsymbol{x}_{\boldsymbol{h}}^{t} \hat{\boldsymbol{\theta}}\right) & =\widehat{\operatorname{Var}}\left(Y_{h}-\hat{\alpha}_{M Q}-\hat{\beta}_{M Q} x_{h}\right) \\
& =\hat{\sigma}_{M Q}^{2}\left(1+\frac{1}{n}+\frac{\left(x_{h}-\bar{x}\right)^{2}}{\sum_{i=1} n\left(x_{i}-\bar{x}\right)^{2}}\right)
\end{aligned}
$$

em que $\left(x_{1}, \ldots, x_{n}\right)$ são os valores observados de $X_{i}, i=1, \ldots, n, \bar{x}=n^{-1} \sum_{i=1}^{n} x_{i} \mathrm{e}$ $\hat{\sigma}_{M Q}$ é o estimador de mínimos quadrados de $\sigma$ sob o modelo usual.

Considere agora $Y_{h}$ uma nova informação da variável aleatória $Y \mid X \in\left(x_{h 1}, x_{h 2}\right]$ independente das demais. Nestas condições, iremos definir o intervalo de previsão aproximado para $Y_{h}$ como

$$
(L I, L S)
$$


em que

$$
\begin{gathered}
L I=\min \left(L I_{x_{h 1}}, L S_{x_{h 1}}, L I_{x_{h 2}}, L S_{x_{h 2}}\right), \\
L S=\max \left(L I_{x_{h 1}}, L S_{x_{h 1}}, L I_{x_{h 2}}, L S_{x_{h 2}}\right), \\
L I_{x_{h 1}}=\boldsymbol{x}_{\boldsymbol{h 1}}^{t} \hat{\boldsymbol{\theta}}^{*}-z_{0,975}\left(\widehat{\operatorname{Var}}\left(Y_{h}-\boldsymbol{x}_{\boldsymbol{h 1}}^{t} \hat{\boldsymbol{\theta}}^{*}\right)\right)^{1 / 2}, \\
L S_{x_{h 1}}=\boldsymbol{x}_{\boldsymbol{h 1}}^{t} \hat{\boldsymbol{\theta}}^{\boldsymbol{*}}+z_{0,975}\left(\widehat{\operatorname{Var}}\left(Y_{h}-\boldsymbol{x}_{\boldsymbol{h 1} \mathbf{\boldsymbol { \theta }}}^{t} \hat{\boldsymbol{\theta}}^{*}\right)\right)^{1 / 2}, \\
L I_{x_{h 2}}=\boldsymbol{x}_{\boldsymbol{h} \mathbf{2}}^{t} \hat{\boldsymbol{\theta}}^{*}-z_{0,975}\left(\widehat{\operatorname{Var}}\left(Y_{h}-\boldsymbol{x}_{\boldsymbol{h} \mathbf{2}}^{t} \hat{\boldsymbol{\theta}}^{*}\right)\right)^{1 / 2}, \\
L S_{x_{h 2}}=\boldsymbol{x}_{\boldsymbol{h 2} \mathbf{\boldsymbol { \theta }}}^{t}+z_{0,975}\left(\widehat{\operatorname{Var}}\left(Y_{h}-\boldsymbol{x}_{\boldsymbol{h} \mathbf{2}}^{t} \hat{\boldsymbol{\theta}}^{*}\right)\right)^{1 / 2},
\end{gathered}
$$

$z_{0,975}$ é o percentil 97,5 de uma distribuição normal padrão, $\boldsymbol{x}_{\boldsymbol{h 1}}^{t}=\left(\begin{array}{ll}1 & x_{h 1}\end{array}\right), \boldsymbol{x}_{\boldsymbol{h 2}}^{t}=$ $\left(1 x_{h 2}\right), \hat{\boldsymbol{\theta}}^{*}=\left(\hat{\alpha}_{M V} \hat{\beta}_{M V}\right)^{t}$, e o estimador de $\operatorname{Var}\left(Y_{h}-\boldsymbol{x}_{\boldsymbol{h}}^{t} \hat{\boldsymbol{\theta}}^{*}\right)$ é dado por

$$
\widehat{\operatorname{Var}}\left(Y_{h}-\boldsymbol{x}_{\boldsymbol{h}}^{t} \hat{\boldsymbol{\theta}}^{*}\right)=\hat{\sigma}_{M V}+\boldsymbol{x}_{\boldsymbol{h}}^{t} \hat{\boldsymbol{\Sigma}}_{\alpha \beta} \boldsymbol{x}_{\boldsymbol{h}}
$$

em que

$$
\hat{\boldsymbol{\Sigma}}_{\alpha \beta}=\left(\begin{array}{cc}
\hat{\sigma}_{\alpha}^{2} & \hat{\sigma}_{\alpha \beta} \\
\hat{\sigma}_{\alpha \beta} & \hat{\sigma}_{\beta}^{2}
\end{array}\right)
$$

é uma estimativa da matriz das covariâncias de $\hat{\alpha}_{M V}$ e $\hat{\beta}_{M V}$.

Nas Figuras 4.1 e 4.2, encontram-se os intervalos de previsão de $Y_{h}$ obtidos sob os dois modelos para cada tom e orelha e para $\left(x_{h_{1}}, x_{h_{2}}\right]$ correspondentes a $(0,5],(5,10], \ldots,(115,120]^{1}$. É possível observar que os intervalos de previsão obtidos sob os dois modelos são similares na maioria dos casos, sendo que os intervalos de previsão obtidos sob o modelo proposto são um pouco mais conservadores do que os intervalos de previsão do modelo usual. Para a orelha direita e o tom de freqüência de $4,0 \mathrm{kHz}$, os intervalos diferem um pouco mais, esse é o caso em que encontramos maiores diferenças entre as estimativas de $\alpha$ e $\beta$ obtidos sob os dois modelos.

\footnotetext{
${ }^{1}$ Os valores máximos de RAEE são: 117, 121, 119 e 118 dBNA, para os tons de $0,5,1,0,2,0$ e $4,0 \mathrm{kHz}$, respectivamente. Utilizamos o intervalo $(115,120]$ ], apenas para facilitar a visualização dos limites de previsão do modelo proposto nos gráficos
} 
Figura 4.1: Ajustes e limites de previsão do modelo usual e do modelo proposto para as frequiências de 0,5 e $1,0 \mathrm{kHz}$.

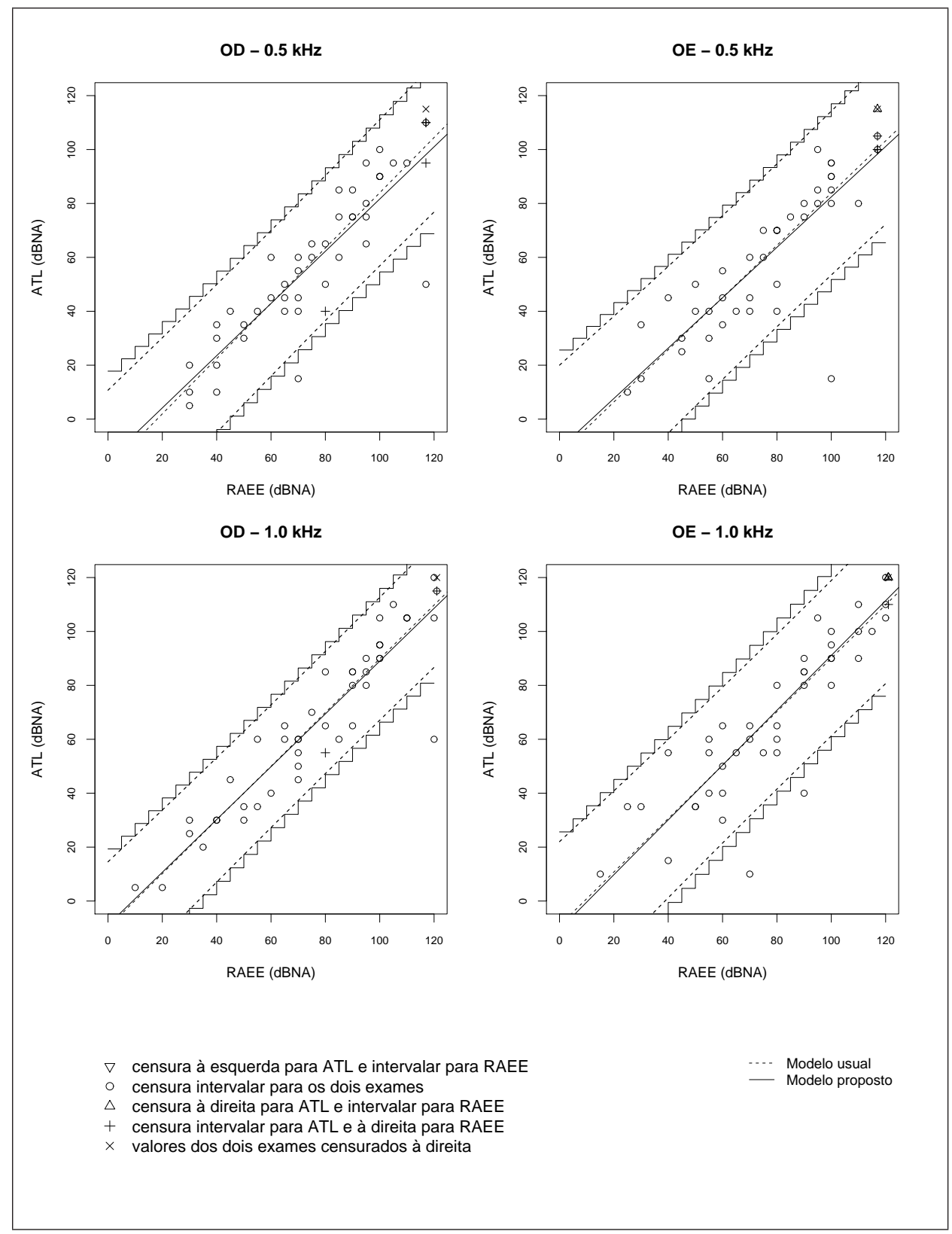


Figura 4.2: Ajustes e limites de previsão do modelo usual e do modelo proposto para as freqüências de 2,0 e 4,0 kHz.

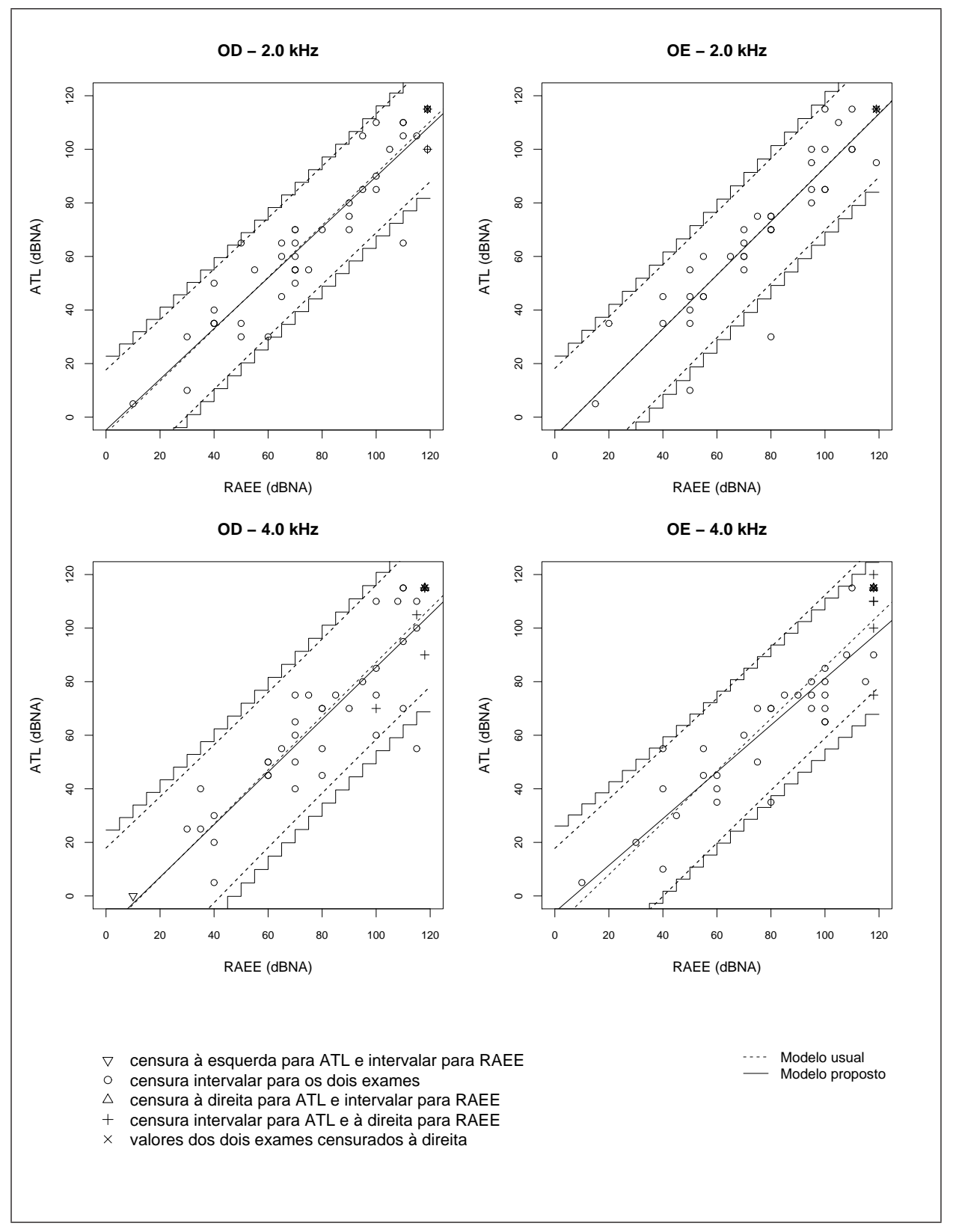




\section{Capítulo 5}

\section{Simulações}

As simulações para comparar os estimadores sob os dois modelos foram realizadas a partir dos seguintes valores para os parâmetros (utilizamos a mesma notação da Equação 2.1):

- $n=\{50,100,500\}$

- $\alpha=-15, \beta=1, \sigma=20$ e $\sigma_{x}=30$;

- $\mu_{x}=\{0,30,60,90,117\}$

Para cada combinação dos valores de $n$ e $\mu_{x}$, foram obtidas 500 amostras de acordo com os seguintes passos:

1. Foram gerados valores de $X_{i}\left(x_{i}\right)$, assumindo que $X_{i} \sim N\left(\mu_{x}, \sigma_{x}^{2}\right), i=$ $1, \ldots, n$.

2. Foram gerados valores de $\epsilon_{i}\left(e_{i}\right), i=1, \ldots, n$, assumindo que $\epsilon_{i} \sim N\left(0, \sigma^{2}\right)$.

3. Foram calculados os valores de $y_{i}=\alpha+\beta x_{i}+e_{i}, i=1, \ldots, n$.

4. Foram construídos intervalos de censura dos valores de $x_{i}, i=1, \ldots, n$, a saber $\left\{\boldsymbol{b}_{\boldsymbol{i}} \in B \mid x_{i} \in \boldsymbol{b}_{\boldsymbol{i}}\right\} \operatorname{com} B=\{(-\infty ; 0],(0 ; 5],(5 ; 10], \ldots,(115 ; 117]$, $(117 ;+\infty)\}$. 
5. Foram obtidos os intervalos de censura dos valores de $y_{i}(i=1, \ldots, n)$. Para obter estes intervalos, considere o conjunto de intervalos dados por $C=$ $\{(-\infty ; 0],(0 ; 5],(5 ; 10], \ldots,(115 ;+\infty)\}$. O intervalo de censura $\boldsymbol{c}_{\boldsymbol{i}}$ de $y_{i}$ é dado por: $\left\{c_{i} \in C \mid x_{i} \in c_{i}\right\}$;

6. Foram definidos como valores censurados de $x_{i}$ e de $y_{i}(i=1, \ldots, n)$, o limite superior do intervalo de censura, quando o limite superior era finito, e o limite inferior do intervalo de censura em caso contrário. Denominamos estes valores de $x_{i}^{*}$ e $y_{i}^{*}$;

7. Foram estimados os parâmetros $\alpha, \beta$ e $\sigma$ através de um modelo de regressão linear simples usual, utilizando os valores originais $y_{i}$ e $x_{i}$;

8. Foram estimados os parâmetros $\alpha, \beta$ e $\sigma$ através de um modelo de regressão linear simples usual, utilizando os valores censurados $y_{i}^{*}$ e $x_{i}^{*}$;

9. Foram estimados os parâmetros $\alpha, \beta$ e $\sigma$ através do modelo proposto, utilizando os intervalos de censura de $y_{i}$ e de $x_{i}$.

Nas Figuras 5.1, 5.2 e 5.3 podem ser vistos os "boxplots" para as estimativas de $\alpha, \beta, \sigma$ obtidas sob o modelo usual utilizando os dados originais $y_{i}$ e $x_{i}$ (MQ), sob o modelo usual utilizando os dados censurados $y_{i}^{*}$ e $x_{i}^{*}$ (MQ*) e sob o modelo proposto utilizando os intervalos de censura (MV). Na Figura 5.4, encontram-se os "boxplots" para as estimativas de $\mu_{x}$ e $\sigma_{x}$ utilizando o modelo proposto (MV).

Seja $\hat{\psi}_{j}$ o valor da estimativa de um parâmetro $\psi$ para uma amostra $j$ de tamanho $n$. O viés de cada estimador foi calculado como:

$$
\text { Viés }=\sum_{j=1}^{500} \frac{\hat{\psi}_{j}}{500}-\psi
$$

$\mathrm{O}$ viés dos estimadores MQ, $\mathrm{MQ}^{*}$ e $\mathrm{MV}$, de $\alpha, \beta$ e $\sigma$ e os respectivos desviospadrão, podem ser vistos na Tabela 5.1 para as amostras de tamanho $n=50,100$ e 500. O viés dos estimadores do modelo proposto (MV), de $\mu_{x}$ e $\sigma_{x}$, podem ser vistos na Tabela 5.2. 
Figura 5.1: "Boxplots" para $\hat{\alpha}$ obtido sob o modelo usual utilizando os dados originais (MQ), sob o modelo usual utilizando os dados censurados (MQ*) e sob o modelo proposto utilizando os intervalos de censura (MV) para $n=50$

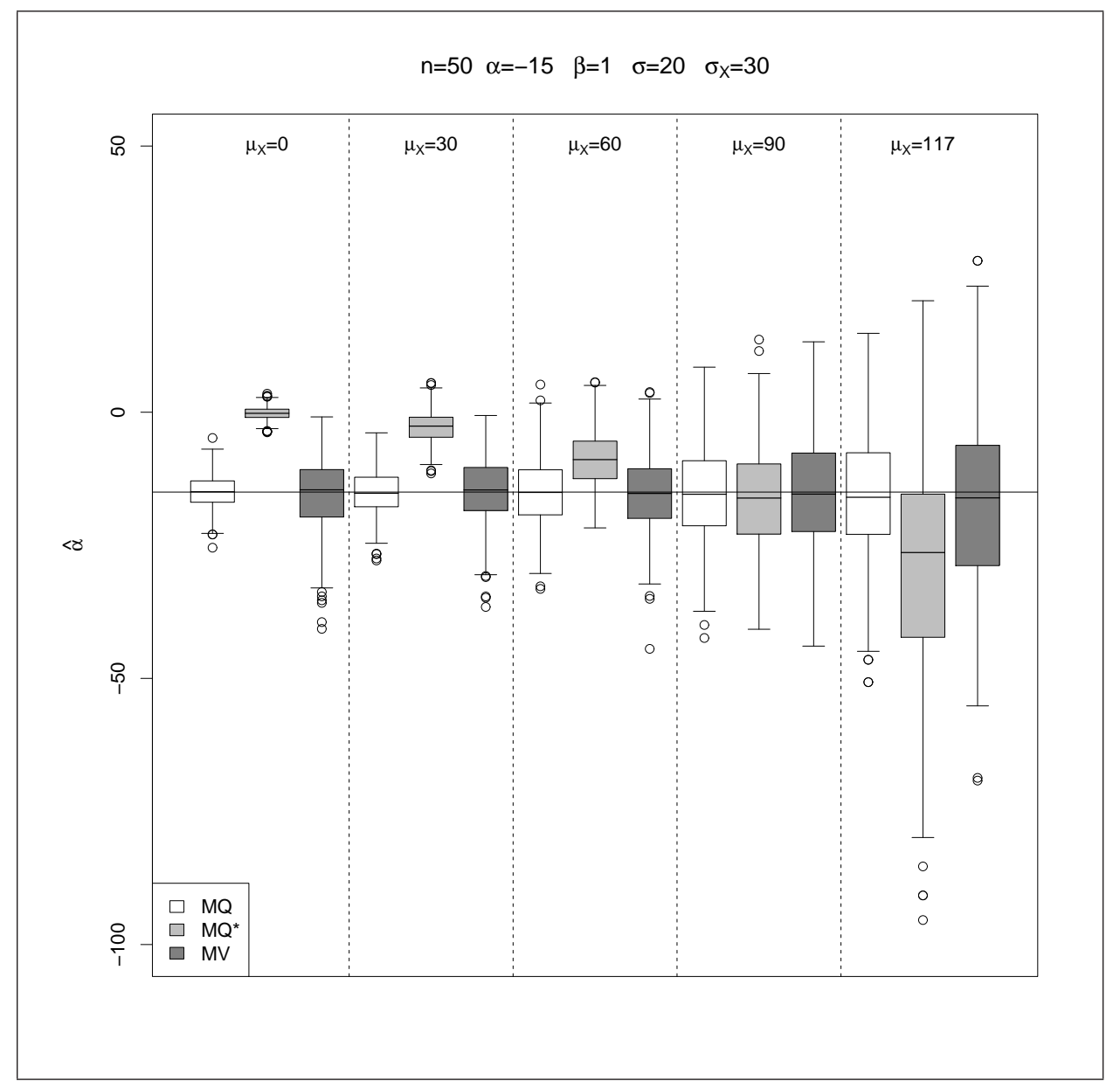


Figura 5.2: "Boxplots" para $\hat{\beta}$ obtido sob o modelo usual utilizando os dados originais (MQ), sob o modelo usual utilizando os dados censurados (MQ*) e sob o modelo proposto utilizando os intervalos de censura (MV) para $n=50$

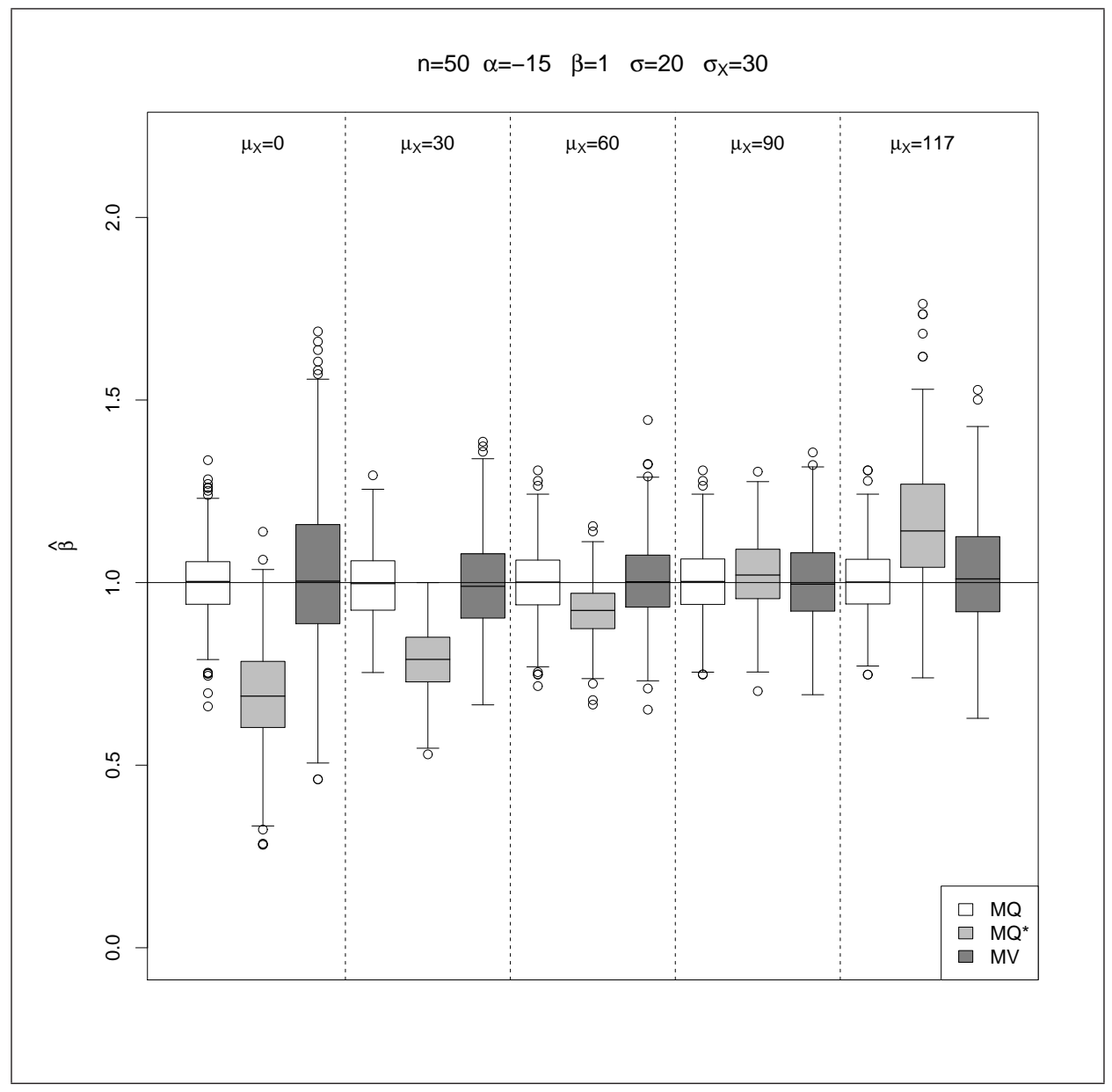


Figura 5.3: "Boxplots" para $\hat{\sigma}$ obtido sob o modelo usual utilizando os dados originais (MQ), sob o modelo usual utilizando os dados censurados (MQ*) e sob o modelo proposto utilizando os intervalos de censura (MV) para $n=50$

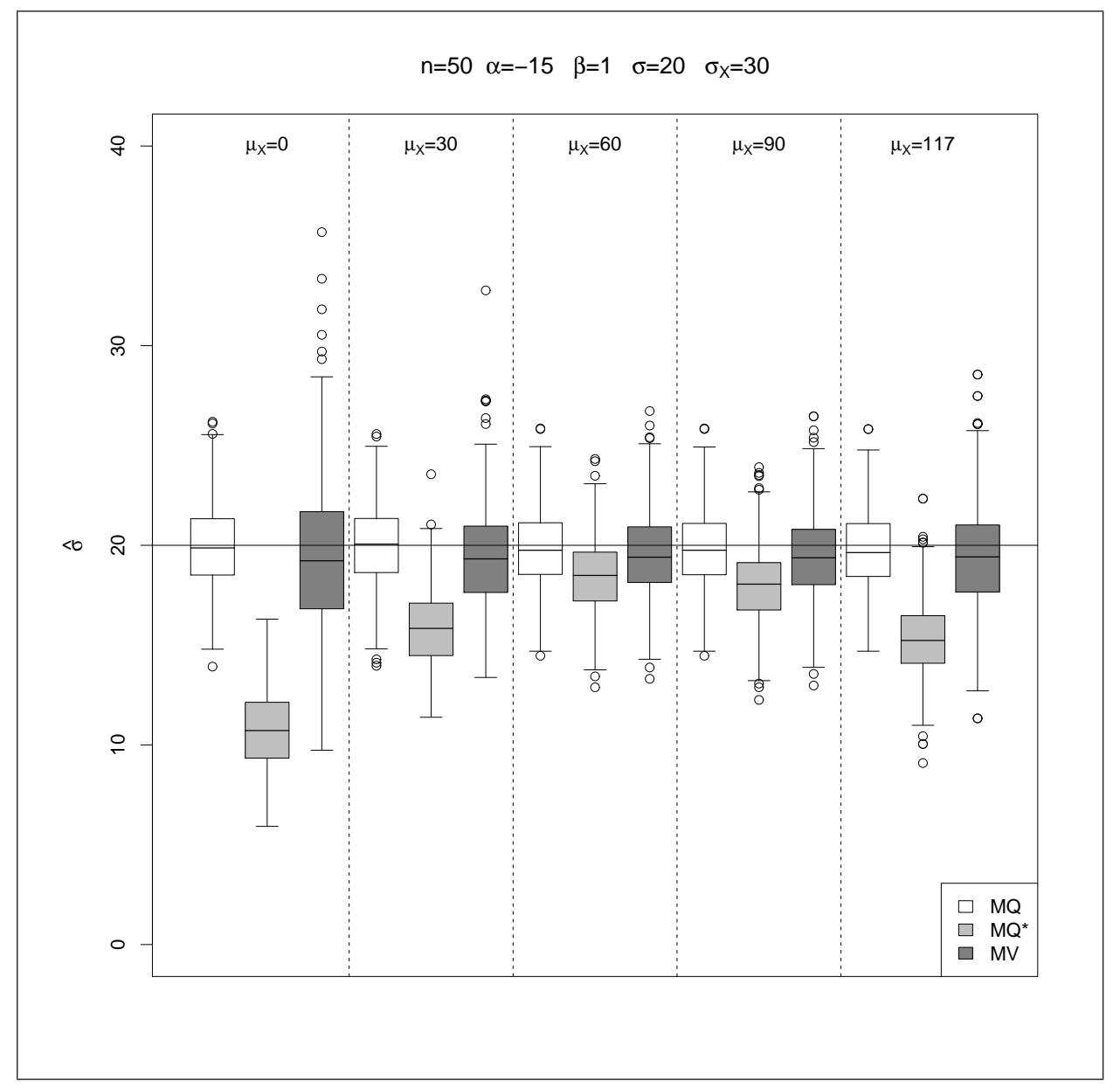


Figura 5.4: "Boxplots" para $\hat{\mu}_{x}$ e $\hat{\sigma}_{x}$ obtidas sob o modelo proposto (MV) para $n=50$

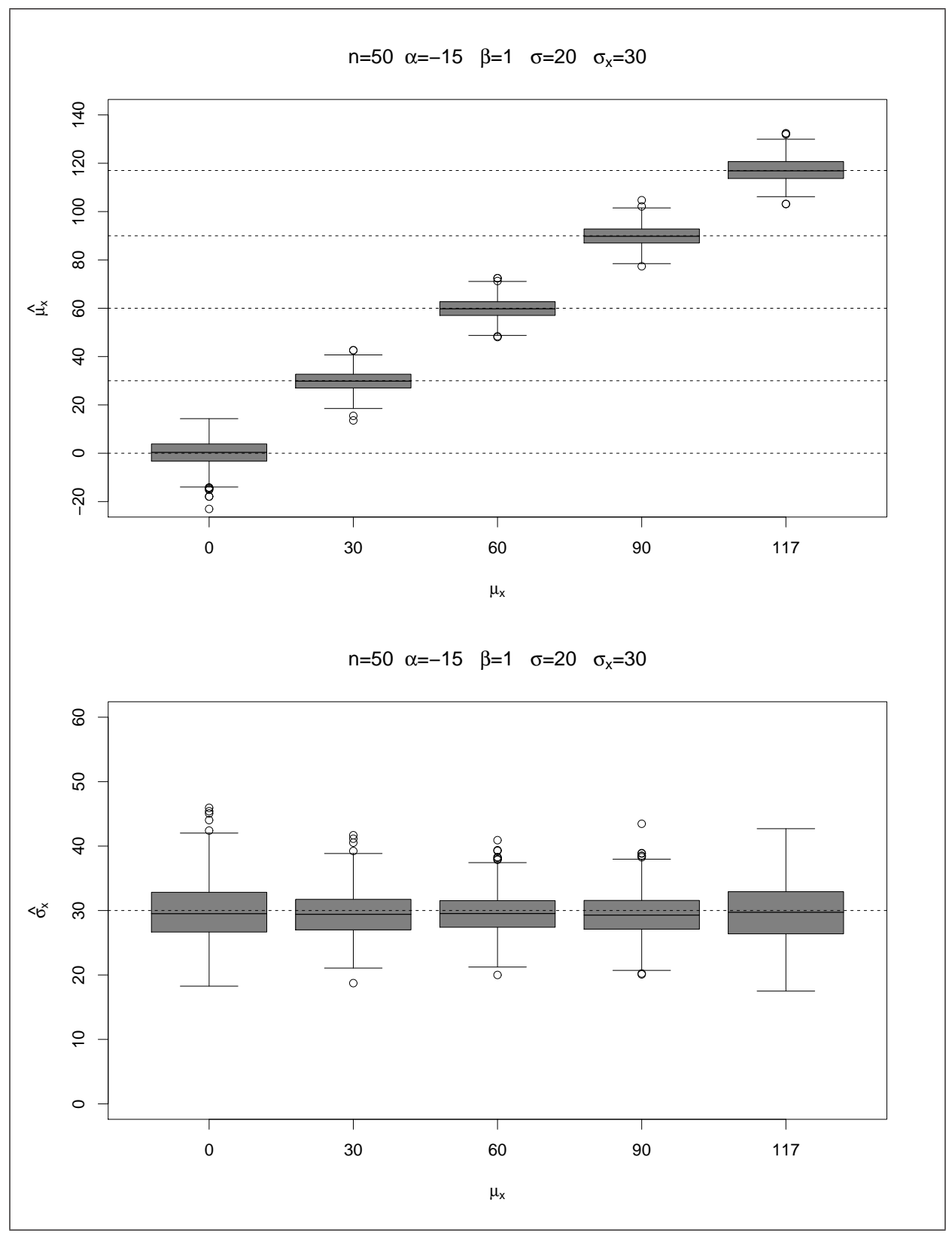


Tabela 5.1: Viés (Desvio-padrão do viés) de $\hat{\alpha}, \hat{\beta}$ e $\hat{\sigma}$ sob o modelo usual utilizando os dados originais (MQ), sob o modelo $\underline{\text { usual utilizando os dados censurados }\left(\mathrm{MQ}^{*}\right) \text { e sob o modelo proposto utilizando os intervalos de censura (MV) }}$

\begin{tabular}{|c|c|c|c|c|c|c|c|c|c|c|}
\hline \multirow{2}{*}{$\mathrm{n}$} & \multirow{2}{*}{$\mu_{x}$} & \multicolumn{3}{|c|}{$\hat{\alpha}$} & \multicolumn{3}{|c|}{$\hat{\beta}$} & \multicolumn{3}{|c|}{$\hat{\sigma}$} \\
\hline & & MQ & MQ* & MV & MQ & MQ* & MV & MQ & $\mathrm{MQ}^{*}$ & MV \\
\hline \multirow{5}{*}{50} & 0 & $0(3)$ & $15(1)$ & $-1(7)$ & $0,00(0,10)$ & $-0,31(0,14)$ & $0,02(0,21)$ & $0(2)$ & $-9(2)$ & $-1(4)$ \\
\hline & 30 & $0(4)$ & $12(3)$ & $0(6)$ & $0,00(0,10)$ & $-0,21(0,09)$ & $-0,01(0,13)$ & $0(2)$ & $-4(2)$ & $-1(3)$ \\
\hline & 60 & $0(6)$ & $6(5)$ & $0(7)$ & $0,00(0,10)$ & $-0,08(0,08)$ & $0,00(0,11)$ & $0(2)$ & $-2(2)$ & $0(2)$ \\
\hline & 90 & $0(9)$ & $-1(10)$ & $-1(10)$ & $0,00(0,10)$ & $0,02(0,10)$ & $0,01(0,12)$ & $0(2)$ & $-2(2)$ & $-1(2)$ \\
\hline & 117 & $-1(11)$ & $-14(19)$ & $-3(16)$ & $0,01(0,09)$ & $0,16(0,17)$ & $0,02(0,15)$ & $0(2)$ & $-5(2)$ & $-1(3)$ \\
\hline \multirow{5}{*}{100} & 0 & $0(2)$ & $15(1)$ & $0(4)$ & $0,00(0,07)$ & $-0,32(0,10)$ & $0,01(0,14)$ & $0(1)$ & $-9(1)$ & $-1(3)$ \\
\hline & 30 & $0(3)$ & $12(2)$ & $0(4)$ & $-0,01(0,07)$ & $-0,21(0,06)$ & $-0,01(0,09)$ & $0(1)$ & $-4(1)$ & $0(2)$ \\
\hline & 60 & $0(4)$ & $6(4)$ & $0(5)$ & $0,00(0,07)$ & $-0,08(0,06)$ & $0,00(0,08)$ & $0(2)$ & $-1(1)$ & $0(2)$ \\
\hline & 90 & $0(6)$ & $-1(7)$ & $-1(7)$ & $0,00(0,07)$ & $0,02(0,07)$ & $0,00(0,08)$ & $0(1)$ & $-2(1)$ & $0(2)$ \\
\hline & 117 & $0(8)$ & $-12(13)$ & $-1(12)$ & $0,00(0,07)$ & $0,15(0,12)$ & $0,01(0,11)$ & $0(1)$ & $-5(1)$ & $0(2)$ \\
\hline \multirow{5}{*}{500} & 0 & $0(1)$ & $15(0)$ & $0(2)$ & $0,00(0,03)$ & $-0,31(0,04)$ & $0,00(0,06)$ & $0(1)$ & $-9(1)$ & $0(1)$ \\
\hline & 30 & $0(1)$ & $12(1)$ & $0(2)$ & $0,00(0,03)$ & $-0,20(0,03)$ & $0,00(0,04)$ & $0(1)$ & $-4(1)$ & $0(1)$ \\
\hline & 60 & $0(2)$ & $6(2)$ & $0(2)$ & $0,00(0,03)$ & $-0,08(0,02)$ & $0,00(0,03)$ & $0(1)$ & $-2(1)$ & $0(1)$ \\
\hline & 90 & $0(3)$ & $-1(3)$ & $0(3)$ & $0,00(0,03)$ & $0,02(0,03)$ & $0,00(0,03)$ & $0(1)$ & $-2(1)$ & $0(1)$ \\
\hline & 117 & $0(4)$ & $-12(5)$ & $0(5)$ & $0,00(0,03)$ & $0,14(0,05)$ & $0,00(0,04)$ & $0(1)$ & $-5(1)$ & $0(1)$ \\
\hline
\end{tabular}


Tabela 5.2: Viés (Desvio-padrão) de $\hat{\mu}_{x}$ e $\hat{\sigma}_{x}$ sob o modelo proposto (MV)

\begin{tabular}{ccccccccc}
\hline \multirow{2}{*}{$\mu_{x}$} & \multicolumn{2}{c}{$\mathrm{n}=50$} & & \multicolumn{2}{c}{$\mathrm{n}=100$} & & \multicolumn{2}{c}{$\mathrm{n}=500$} \\
\cline { 2 - 3 } \cline { 8 - 9 } \cline { 8 - 9 } & $\hat{\mu}_{x}$ & $\hat{\sigma}_{x}$ & & $\hat{\mu}_{x}$ & $\hat{\sigma}_{x}$ & & $\hat{\mu}_{x}$ & $\hat{\sigma}_{x}$ \\
\hline 0 & $0(5)$ & $0(5)$ & & $0(4)$ & $0(3)$ & & $0(2)$ & $0(1)$ \\
30 & $0(4)$ & $-1(4)$ & & $0(3)$ & $0(2)$ & & $0(2)$ & $0(1)$ \\
60 & $0(4)$ & $0(3)$ & & $0(3)$ & $0(2)$ & & $0(1)$ & $0(1)$ \\
90 & $0(4)$ & $-1(3)$ & & $0(3)$ & $0(2)$ & & $0(1)$ & $0(1)$ \\
117 & $0(5)$ & $0(5)$ & & $0(4)$ & $0(3)$ & & $0(2)$ & $0(1)$ \\
\hline
\end{tabular}

Nas simulações realizadas, os estimadores $\mathrm{MV}$ de $\alpha, \beta$ e $\sigma$ apresentaram um pequeno viés para amostras de tamanho $n=50$. Nota-se, que para este tamanho amostral, o estimador MV de $\sigma$ apresenta viés negativo de 1 unidade, em quase todos os casos. $\mathrm{O}$ viés dos estimadores $\mathrm{MV}$ dos parâmetros $\alpha, \beta$ e $\sigma$ é quase nulo para amostras de tamanho $n=100$ e praticamente inexistente para amostras de tamanho $n=500$. Já os estimadores $\mathrm{MQ}^{*}$ de $\alpha, \beta$ e $\sigma$ apresentam valores enviesados independentemente do tamanho amostral. O viés do estimador MQ* de $\alpha$ é positivo para pequenos valores de $\mu_{x}$ e negativo para valores maiores de $\mu_{x}$. O estimador $\mathrm{MQ}^{*}$ de $\beta$ é negativo para pequenos valores de $\mu_{x}$ e positivo para valores maiores de $\mu_{x}$. Por outro lado o estimador $\mathrm{MQ}^{*}$ de $\sigma$ sempre apresenta valores enviesados negativamente, sendo o viés maior (em valores absolutos) para os casos extremos, $\mu_{x}=0$ e $\mu_{x}=117$.

Pode-se notar que a variabilidade dos estimadores MQ* de $\alpha$ e $\beta$ é, em geral, menor que aquela dos estimadores $\mathrm{MV}$, exceto para $\mu_{x}=117$. A variabilidade do estimador $\mathrm{MQ}^{*}$ de $\sigma$, por sua vez, se mantém constante e, na maioria dos casos, ligeiramente menor que a variabilidade do estimador MV de $\sigma$.

Os estimadores MV de $\mu_{x}$ e $\sigma_{x}$ se mostraram praticamente não enviesados ou com viés de até 1 unidade. 


\section{Capítulo 6}

\section{Discussão}

Investigamos o comportamento dos estimadores de máxima verossimilhança dos parâmetros de um modelo de regressão linear simples em que tanto a variável resposta como a variável independente estão sujeitas a censura intervalar. Mostramos através de simulações que, ao utilizar dados censurados no modelo usual, obtemos estimadores enviesados.

Em alguns casos, é fácil entender o motivo do viés gerado pelos estimadores MQ*. Considere a Figura 6.1. Nela, vemos que os limites de detecção de $Y$ são 0 e 115 e os limites de detecção de $X$ são 0 e 117. Podemos determinar 9 regiões $q_{i}, i=1, \ldots, 9$, baseados nestes limites. Na região $\mathrm{q} 1$, tanto os valores de $X$ como os valores de $Y$ são censurados à esquerda, na região q2, os valores de $X$ são censurados à esquerda e os de $Y$ apresentam censura intervalar, e assim por diante.

Para cada amostra gerada nas simulações calculamos a proporção de observações pertencentes a cada região. O passo seguinte foi calcular a média dessas proporções, considerando as 500 simulações geradas para $n=50$ (Figura 6.2). Observando o caso em que $\mu_{x}=0$, notamos que, em média, $47 \%$ dos pares apresentam censura à esquerda tanto para $X$ como para $Y$. Ao realizar o ajuste pelo método de mínimos quadrados, desconsiderando o fato dos dados serem censurados, todos os valores da região q1 passam a ter os valores $x=0$ e $y=0$. Este "deslocamento" dos pontos da região q1, faz com que o coeficiente angular da 
Figura 6.1: Descrição das regiões q1,..,q9

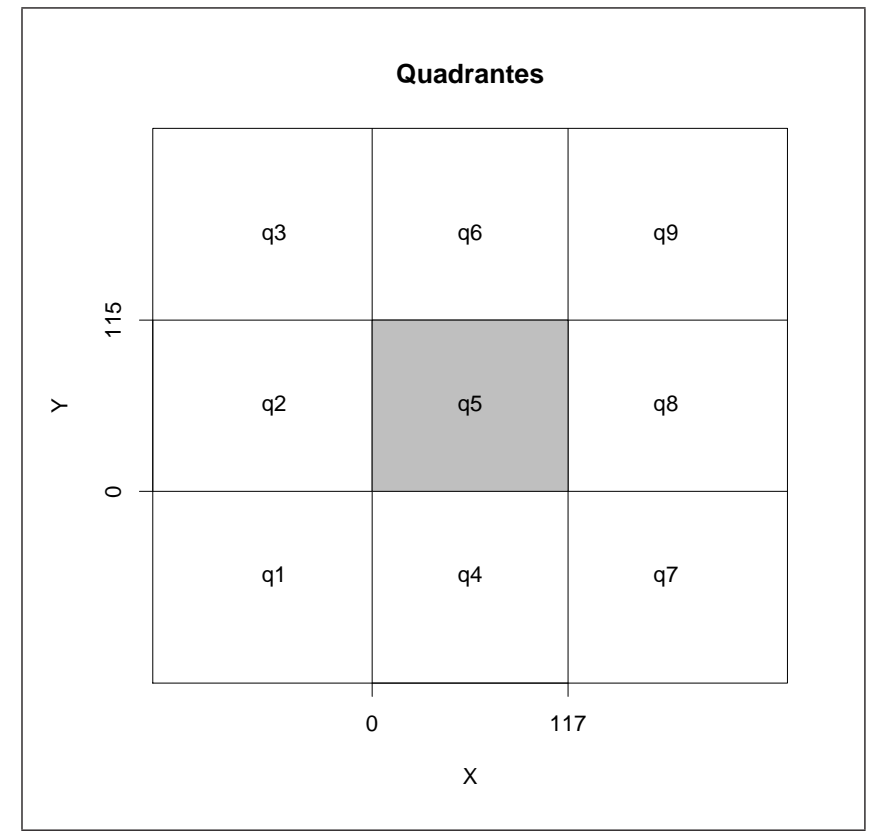

reta seja afetado, o que gera o viés dos estimadores $\hat{\alpha}$ e $\hat{\beta}$. Pelo mesmo motivo, o estimador $\mathrm{MQ}^{*}$ subestima o valor de $\sigma$, pois neste caso, a quantidade de valores iguais ( $x=0$ e $y=0$ ) aumenta. Observe que os casos em que os estimadores MQ* de $\alpha$ e $\beta$ apresentaram-se menos enviesados são aqueles em que $\mu_{x}=60$ e $\mu_{x}=90$. Nesses casos, ocorre uma concentração maior de dados no quadrante q5, em que tanto os valores de $X$ como os valores de $Y$ são censurados de forma intervalar.

Ao contrário dos estimadores MQ*, os estimadores MV de $\alpha$ e $\beta$ apresentaram viés nulo em quase todos os casos considerados. O estimador MV de $\sigma$ apresentou viés negativo. Porém, sabe-se que o estimador de máxima verossimilhança de $\sigma$ (denotado por $\hat{\sigma}$ ) de um modelo de regressão linear simples sem dados censurados é enviesado (Neter et al., 1996).

Utilizando a correção $\hat{\sigma}^{*}=\sqrt{\frac{n}{n-2}} \hat{\sigma}$ para o estimador MV de $\sigma$, encontramos resultados ainda ligeiramente enviesados como pode ser visto na Figura 6.3.

Para análise dos dados de nossas aplicações (ATL e RAEE), os dois modelos apresentaram valores estimados bem próximos. Estes resultados são compatíveis 
Figura 6.2: Distribuição média dos dados nas regiões q1,...,q9 e retas construídas utilizando as médias de $\hat{\alpha}$ e $\hat{\beta}$ obtidas sob o modelo usual utilizando os dados originais (MQ), sob o modelo usual utilizando os dados censurados (MQ*) e sob o modelo proposto utilizando os intervalos de censura (MV)

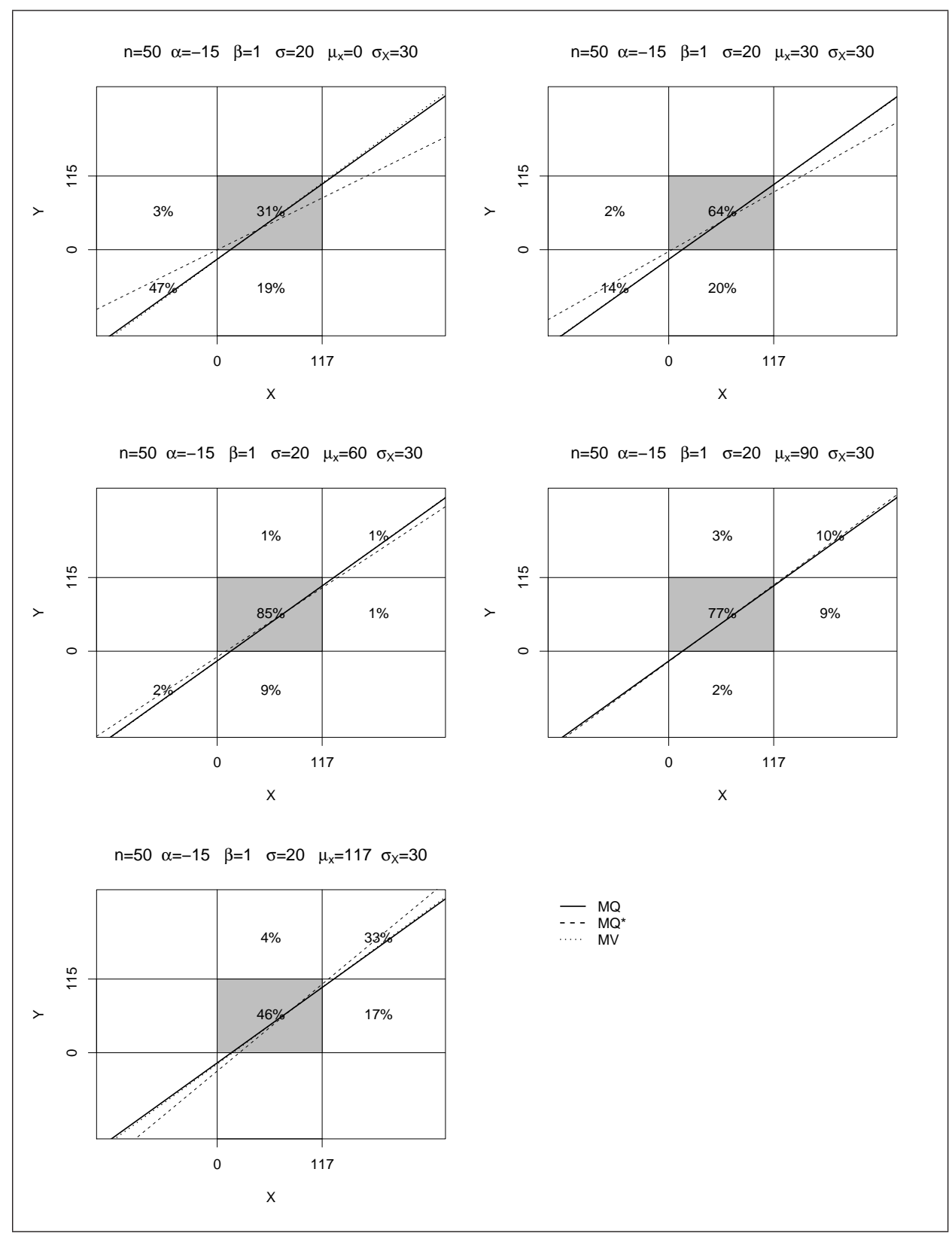


Figura 6.3: "Boxplots" para $\hat{\sigma}$ obtido sob o modelo usual utilizando os dados originais (MQ), sob o modelo usual utilizando os dados censurados (MQ*), sob o modelo proposto utilizando os intervalos de censura (MV) e sob o modelo proposto utilizando os intervalos de censura com correção (MV com correção) para $n=50$

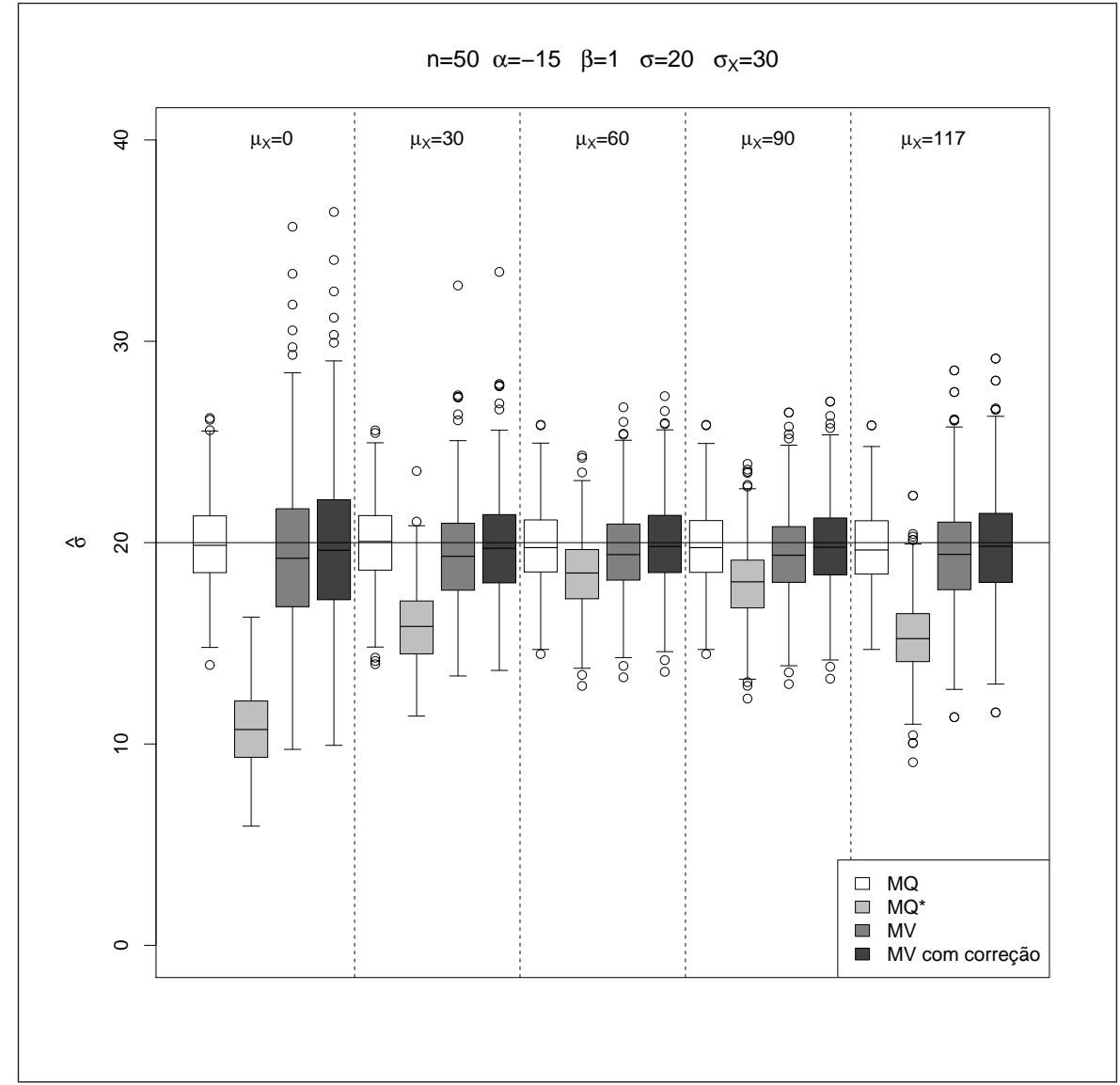


com os resultados das simulações, uma vez que, os dados de RAEE e ATL se concentram na região q5, como pode ser visto nas Figuras 6.4 e 6.5.

Algumas das limitações nos estudos de simulação realizados foram: não simular a censura que ocorre quando há desconforto na realização do exame de RAEE e não simular dados censurados baseados ora nos intervalos do conjunto $C=$ $\{(-\infty, 2], \ldots,(102,105],(107,112],(112,117],(117,+\infty)\}$, que poderiam ocorrer caso o exame de RAEE iniciasse pelo valor de intensidade igual a 117dBNA, ora nos intervalos do conjunto $B=\{(-\infty ; 0],(0 ; 5],(5 ; 10], \ldots,(115 ; 117],(117 ;+\infty)\}$, utilizados quando o exame de RAEE começa por valores de intensidade menores que $117 \mathrm{dBNA}$.

Com relação ao diagnóstico do modelo proposto, nos limitamos à análise descritiva dos resíduos médios (Equação 3.1) e à construção de gráficos de quantis para investigar a normalidade dos resíduos e da variável independente.

Apesar de os resultados indicarem que os estimadores do modelo proposto são não enviesados, estudos futuros são necessários para investigar o comportamento desses estimadores em casos mais gerais. Além disso, há necessidade do desenvolvimento das técnicas de diagnóstico e de testes estatísticos para verificar se as suposições de normalidade dos resíduos e da variável independente estão satisfeitas. Algumas opções, são a utilização dos resíduos propostos por Topp e Gomez (2004) para modelos de regressão em que o erro tem distribuição normal e a variável independente é discreta e está sujeita a censura intervalar, além do teste de normalidade proposto por Ren (2003). 
Figura 6.4: Distribuição dos dados de ATL e RAEE nas regiões q1,..,q9 e modelos ajustados para as freqüências de 0,5 e 1,0

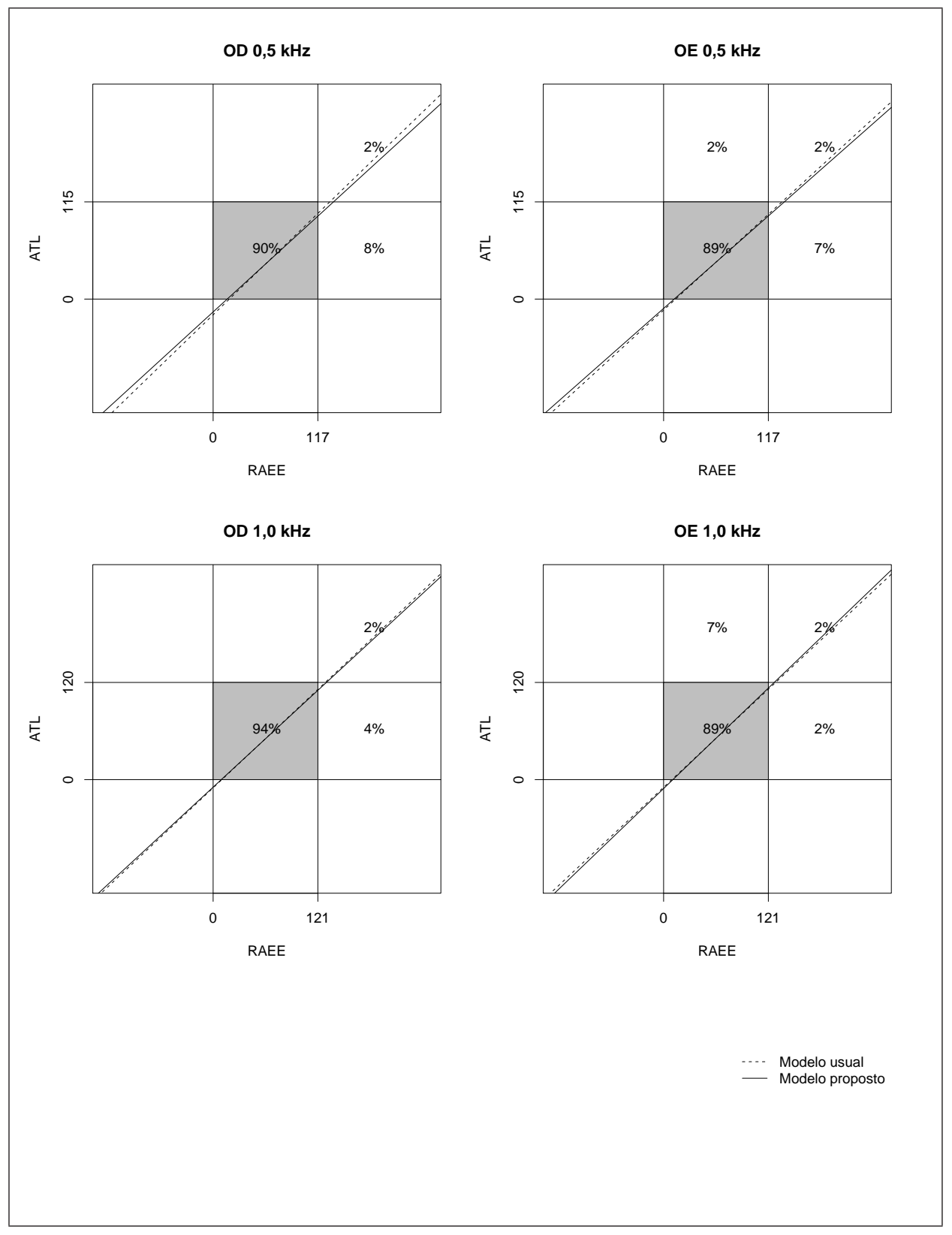


Figura 6.5: Distribuição dos dados de ATL e RAEE nas regiões q1,...,q9 e modelos ajustados para as freqüências de 2,0 e 4,0

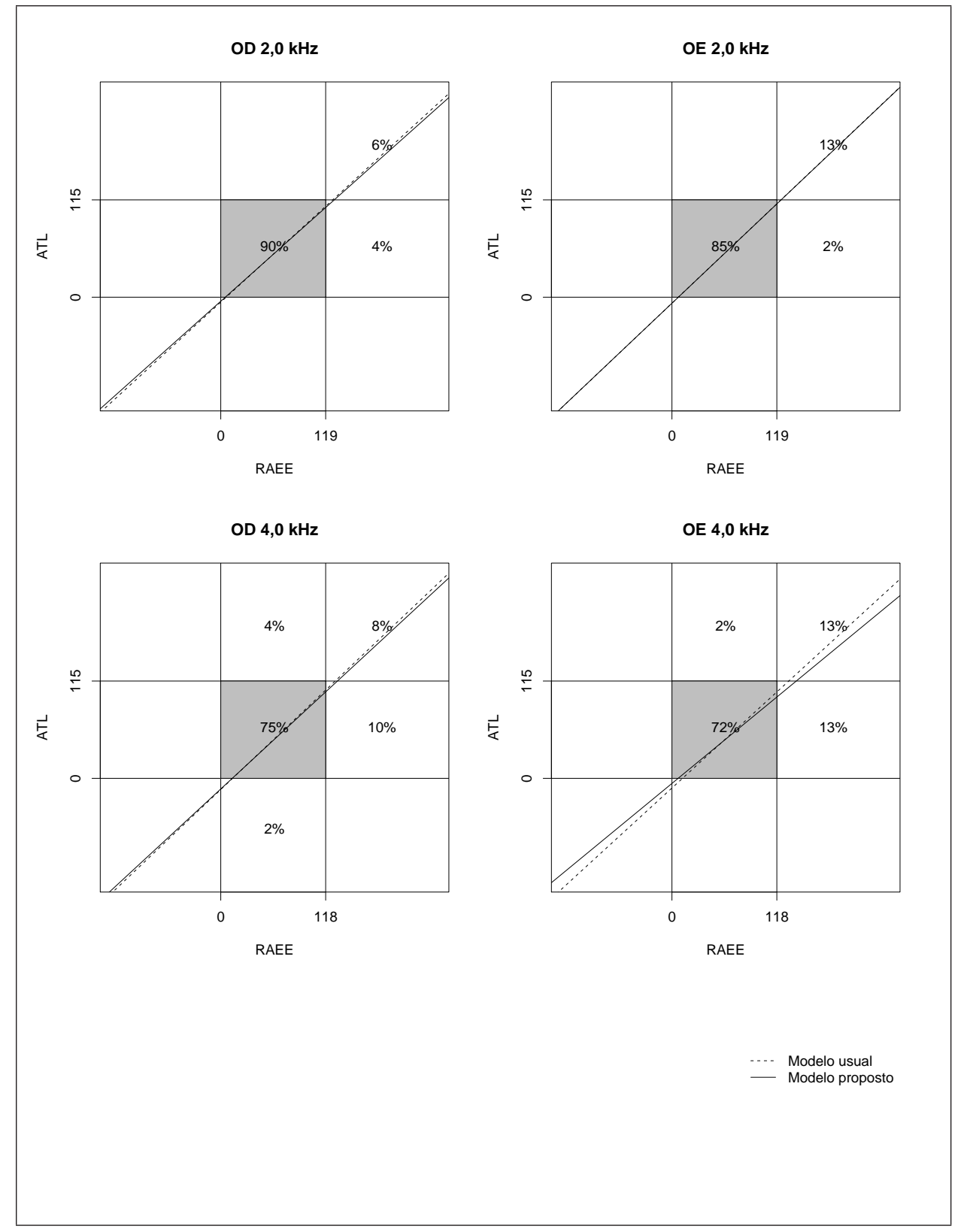





\section{Apêndice A}

\section{Método de Newton-Raphson}

Considere o modelo ( 2.1). Seja, $\dot{\boldsymbol{l}}(\boldsymbol{\theta})=\partial l(\boldsymbol{\theta}) / \partial \boldsymbol{\theta}$, a matriz das expressões das derivadas parciais, e $\ddot{\boldsymbol{l}}(\boldsymbol{\theta})=\partial^{2} l(\boldsymbol{\theta}) / \partial \boldsymbol{\theta} \partial \boldsymbol{\theta}^{T}$ a matriz Hessiana, da função de verossimilhança (2.4). Seja

$$
x_{i}^{*}=\left\{\begin{array}{l}
x_{\text {inf }}, \text { se } x_{\text {sup }} \text { não é finito } \\
x_{\text {sup }}, \text { se } x_{\text {sup }}<+\infty
\end{array}\right.
$$

e

$$
y_{i}^{*}=\left\{\begin{array}{l}
y_{\text {inf }_{i}}, \text { se } y_{\text {sup }_{i}} \text { não é finito } \\
y_{\text {sup }_{i}}, \text { se } y_{\text {sup }_{i}}<+\infty
\end{array}\right.
$$

para $i=1, \ldots, n$. Para encontrar os valores das estimativas de máxima verossimilhança de $\boldsymbol{\theta}$, utilizamos o seguinte algoritmo:

1. Obter uma estimativa inicial de $\boldsymbol{\theta}^{\mathbf{0}}, \hat{\boldsymbol{\theta}}^{\mathbf{0}}=\left(\hat{\alpha}^{0}, \hat{\beta}^{0}, \hat{\sigma}^{0}, \hat{\mu}_{x}^{0}, \hat{\sigma}_{x}^{0}\right)^{T}$. Para obter as estimativas iniciais $\hat{\alpha}^{0}, \hat{\beta}^{0}$ e $\hat{\sigma}^{0}$, utilizamos um modelo de regressão linear simples entre $y_{i}^{*}$ e $x_{i}^{*}$. Para encontrar as estimativas $\hat{\mu}_{x}^{0}$ e $\hat{\sigma}_{x}^{0}$, utilizamos a média e o desvio-padrão amostral de $x_{i}^{*}$;

2. Calcular $\dot{\boldsymbol{l}}\left(\hat{\boldsymbol{\theta}}^{\mathbf{0}}\right)$ e $\ddot{\boldsymbol{l}}\left(\hat{\boldsymbol{\theta}}^{\mathbf{0}}\right)$;

3. Calcular $\hat{\boldsymbol{\theta}}^{i+1}=\hat{\boldsymbol{\theta}}^{i}-\dot{\boldsymbol{l}}\left(\hat{\boldsymbol{\theta}}^{i}\right) \ddot{\boldsymbol{l}}\left(\hat{\boldsymbol{\theta}}^{i}\right)^{-1}$; 
4. Calcular erro $=\max \left(\left|\hat{\alpha}^{i+1}-\hat{\alpha}^{i}\right|,\left|\hat{\beta}^{i+1}-\hat{\beta}^{i}\right|,\left|\hat{\sigma}^{i+1}-\hat{\sigma}^{i}\right|,\left|\hat{\mu}_{x}^{i+1}-\hat{\mu}_{x}^{i}\right|\left|\hat{\sigma}_{x}^{i+1}-\hat{\sigma}_{x}^{i}\right|\right)$;

5. Enquanto o erro $>$ eps e $i \leq n_{i t}$ (eps representa o erro utilizado como critério de convergência e $n_{i t}$ representa o número máximo de iterações permitidas $^{1}$ ) repetir os passos 3 e 4 ;

6. Retornar como estimativas de máxima verossimilhança de $\boldsymbol{\theta}, \hat{\boldsymbol{\theta}}=\hat{\boldsymbol{\theta}}^{i+1}$ e como matriz de covariância estimada de $\hat{\boldsymbol{\theta}}$, a matriz $\hat{\boldsymbol{\Sigma}}=\left(\ddot{\boldsymbol{l}}\left(\hat{\boldsymbol{\theta}}^{i+1}\right)\right)^{-1}$;

7. Se erro > eps retornar a mensagem "Não ocorreu convergência!!!".

\footnotetext{
${ }^{1}$ Se não forem definidos, serão considerados os valores eps $=0,001$ e $n_{i t}=20$.
} 


\section{Apêndice B}

\section{Expressões para o vetor score e matriz Hessiana de $l(\theta)$}

As expressões do vetor score, $\dot{\boldsymbol{l}}(\boldsymbol{\theta})$ e da matrix Hessiana, $\ddot{\boldsymbol{l}}(\boldsymbol{\theta})$ são

$$
\dot{\boldsymbol{l}}(\boldsymbol{\theta})=\left(\begin{array}{c}
\frac{\partial l(\boldsymbol{\theta})}{\partial \alpha} \\
\frac{\partial l(\boldsymbol{\theta})}{\partial \beta} \\
\frac{\partial l(\boldsymbol{\theta})}{\partial \sigma} \\
\frac{\partial l(\boldsymbol{\theta})}{\partial \mu_{x}} \\
\frac{\partial l(\boldsymbol{\theta})}{\partial \sigma_{x}}
\end{array}\right)
$$

$\mathrm{e}$

$$
\ddot{\boldsymbol{l}}(\boldsymbol{\theta})=\left[\begin{array}{lllll}
\frac{\partial^{2} l(\boldsymbol{\theta})}{\partial \alpha^{2}} & \frac{\partial^{2} l(\boldsymbol{\theta})}{\partial \alpha \partial \beta} & \frac{\partial^{2} l(\boldsymbol{\theta})}{\partial \alpha \partial \sigma} & \frac{\partial^{2} l(\boldsymbol{\theta})}{\partial \alpha \partial \mu_{x}} & \frac{\partial^{2} l(\boldsymbol{\theta})}{\partial \alpha \partial \sigma_{x}} \\
\frac{\partial^{2} l(\boldsymbol{\theta})}{\partial \alpha \partial \beta} & \frac{\partial^{2} l(\boldsymbol{\theta})}{\partial \beta^{2}} & \frac{\partial^{2} l(\boldsymbol{\theta})}{\partial \beta \partial \sigma} & \frac{\partial^{2} l(\boldsymbol{\theta})}{\partial \beta \partial \mu_{x}} & \frac{\partial^{2} l(\boldsymbol{\theta})}{\partial \beta \partial \sigma_{x}} \\
\frac{\partial^{2} l(\boldsymbol{\theta})}{\partial \alpha \partial \sigma} & \frac{\partial^{2} l(\boldsymbol{\theta})}{\partial \beta \partial \sigma} & \frac{\partial^{2} l(\boldsymbol{\theta})}{\partial \sigma^{2}} & \frac{\partial^{2} l(\boldsymbol{\theta})}{\partial \sigma \partial \mu_{x}} & \frac{\partial^{2} l(\boldsymbol{\theta})}{\partial \sigma \partial \sigma_{x}} \\
\frac{\partial^{2} l(\boldsymbol{\theta})}{\partial \alpha \partial \mu_{x}} & \frac{\partial^{2} l(\boldsymbol{\theta})}{\partial \beta \partial \mu_{x}} & \frac{\partial^{2} l(\boldsymbol{\theta})}{\partial \sigma \partial \mu_{x}} & \frac{\partial^{2} l(\boldsymbol{\theta})}{\partial \mu_{x}^{2}} & \frac{\partial^{2} l(\boldsymbol{\theta})}{\partial \mu_{x} \partial \sigma_{x}} \\
\frac{\partial^{2} l(\boldsymbol{\theta})}{\partial \alpha \partial \sigma_{x}} & \frac{\partial^{2} l(\boldsymbol{\theta})}{\partial \beta \partial \sigma_{x}} & \frac{\partial^{2} l(\boldsymbol{\theta})}{\partial \sigma \partial \sigma_{x}} & \frac{\partial^{2} l(\boldsymbol{\theta})}{\partial \mu_{x} \partial \sigma_{x}} & \frac{\partial^{2} l(\boldsymbol{\theta})}{\partial \sigma_{x}^{2}}
\end{array}\right]
$$


Como a função de verossimilhança pode ser escrita na forma:

$$
l(\boldsymbol{\theta})=\sum_{i=1}^{n} l_{i}(\boldsymbol{\theta})
$$

em que:

$$
l_{i}(\boldsymbol{\theta})=\ln \left(\int_{y_{i n f_{i}}}^{y_{s u p_{i}}} \int_{x_{i n f_{i}}}^{x_{\text {sup }_{i}}} f(y \mid x, \alpha, \beta, \sigma) f\left(x \mid \mu_{x}, \sigma_{x}\right) d x d y\right)
$$

podemos escrever as expressões de $\dot{\boldsymbol{l}}(\boldsymbol{\theta})$ e de $\ddot{\boldsymbol{l}}(\boldsymbol{\theta})$ como:

$$
\dot{\boldsymbol{l}}(\boldsymbol{\theta})=\sum_{i=1}^{n}\left(\begin{array}{c}
\frac{\partial l_{i}(\boldsymbol{\theta})}{\partial \alpha} \\
\frac{\partial l_{i}(\boldsymbol{\theta})}{\partial \beta} \\
\frac{\partial l_{i}(\boldsymbol{\theta})}{\partial \sigma} \\
\frac{\partial l_{i}(\boldsymbol{\theta})}{\partial \mu_{x}} \\
\frac{\partial l_{i}(\boldsymbol{\theta})}{\partial \sigma_{x}}
\end{array}\right)
$$

e

$$
\ddot{\boldsymbol{l}}(\boldsymbol{\theta})=\sum_{i=1}^{n}\left[\begin{array}{ccccc}
\frac{\partial^{2} l_{i}(\boldsymbol{\theta})}{\partial \alpha^{2}} & \frac{\partial^{2} l_{i}(\boldsymbol{\theta})}{\partial \alpha \partial \beta} & \frac{\partial^{2} l_{i}(\boldsymbol{\theta})}{\partial \alpha \partial \sigma} & \frac{\partial^{2} l_{i}(\boldsymbol{\theta})}{\partial \alpha \partial \mu_{x}} & \frac{\partial^{2} l_{i}(\boldsymbol{\theta})}{\partial \alpha \partial \sigma_{x}} \\
\frac{\partial^{2} l_{i}(\boldsymbol{\theta})}{\partial \partial \partial \beta} & \frac{\partial^{2} l_{i}(\boldsymbol{\theta})}{\partial \beta^{2}} & \frac{\partial^{2} l_{i}(\boldsymbol{\theta})}{\partial \beta \partial \sigma} & \frac{\partial^{2} l_{i}(\boldsymbol{\theta})}{\partial \beta \partial \mu_{x}} & \frac{\partial^{2} l_{i}(\boldsymbol{\theta})}{\partial \beta \partial \sigma_{x}} \\
\frac{\partial^{2} l_{i}(\boldsymbol{\theta})}{\partial \alpha \partial \sigma} & \frac{\partial^{2} l_{i}(\boldsymbol{\theta})}{\partial \beta \partial \sigma} & \frac{\partial^{2} l_{i}(\boldsymbol{\theta})}{\partial \sigma^{2}} & \frac{\partial^{2} l_{i}(\boldsymbol{\theta})}{\partial \sigma \partial \mu_{x}} & \frac{\partial^{2} l_{i}(\boldsymbol{\theta})}{\partial \sigma \partial \sigma_{x}} \\
\frac{\partial^{2} l_{i}(\boldsymbol{\theta})}{\partial \alpha \partial \mu_{x}} & \frac{\partial^{2} l_{i}(\boldsymbol{\theta})}{\partial \beta \partial \mu_{x}} & \frac{\partial^{2} l_{i}(\boldsymbol{\theta})}{\partial \sigma \partial \mu_{x}} & \frac{\partial^{2} l_{i}(\boldsymbol{\theta})}{\partial \mu_{x}^{2}} & \frac{\partial^{2} l_{i}(\boldsymbol{\theta})}{\partial \mu_{x} \partial \sigma_{x}} \\
\frac{\partial^{2} l_{i}(\boldsymbol{\theta})}{\partial \alpha \partial \sigma_{x}} & \frac{\partial^{2} l_{i}(\boldsymbol{\theta})}{\partial \beta \partial \sigma_{x}} & \frac{\partial^{2} l_{i}(\boldsymbol{\theta})}{\partial \sigma \partial \sigma_{x}} & \frac{\partial^{2} l_{i}(\boldsymbol{\theta})}{\partial \mu_{x} \partial \sigma_{x}} & \frac{\partial^{2} l_{i}(\boldsymbol{\theta})}{\partial \sigma_{x}^{2}}
\end{array}\right]
$$


Para escrever as derivadas de $l_{i}(\boldsymbol{\theta})$, vamos considerar as expressões abaixo:

$$
\phi(x, y)=f(y \mid x, \alpha, \beta, \sigma) f\left(x \mid \mu_{x}, \sigma_{x}\right)
$$

$\mathrm{e}$

$$
\begin{aligned}
& \Phi_{i}(\cdot)=\Phi_{i}\left(y_{\text {inf }_{i}}, y_{\text {sup }_{i}}, x_{\text {inf }_{i}}, x_{\text {sup }_{i}}, \boldsymbol{\theta}\right) \\
& \Phi_{i}(\cdot)=\int_{y_{\text {inf }}}^{y_{\text {sup }_{i}}} \int_{x_{\text {inf }}}^{x_{\text {sup }_{i}}} f(y \mid x, \alpha, \beta, \sigma) f\left(x \mid \mu_{x}, \sigma_{x}\right) d x d y
\end{aligned}
$$

Dessa forma, temos:

$$
\begin{aligned}
& \frac{\partial l_{i}(\boldsymbol{\theta})}{\partial \alpha}=\frac{1}{\Phi_{i}(\cdot)} \int_{y_{i n f_{i}}}^{y_{\text {sup }_{i}}} \int_{x_{\text {inf }}}^{x_{\text {sup }_{i}}} \frac{y-\alpha-\beta x}{\sigma^{2}} \phi(x, y) d x d y \\
& \frac{\partial l_{i}(\boldsymbol{\theta})}{\partial \beta}=\frac{1}{\Phi_{i}(\cdot)} \int_{y_{i n f_{i}}}^{y_{s u p_{i}}} \int_{x_{i n f_{i}}}^{x_{s u p_{i}}} \frac{(y-\alpha-\beta x) x}{\sigma^{2}} \phi(x, y) d x d y \\
& \frac{\partial l_{i}(\boldsymbol{\theta})}{\partial \sigma}=\frac{1}{\Phi_{i}(\cdot)} \int_{y_{i n f_{i}}}^{y_{\text {sup }_{i}}} \int_{x_{\text {inf }}}^{x_{\text {sup }_{i}}}\left(-\frac{1}{\sigma}+\frac{(y-\alpha-\beta x)^{2}}{\sigma^{3}}\right) \phi(x, y) d x d y \\
& \frac{\partial l_{i}(\boldsymbol{\theta})}{\partial \mu_{x}}=\frac{1}{\Phi_{i}(\cdot)} \int_{y_{\text {inf }}}^{y_{\text {sup }_{i}}} \int_{x_{\text {inf }}}^{x_{\text {sup }}} \frac{x-\mu_{x}}{\sigma_{x}^{2}} \phi(x, y) d x d y \\
& \frac{\partial l_{i}(\boldsymbol{\theta})}{\partial \sigma_{x}}=\frac{1}{\Phi_{i}(\cdot)} \int_{y_{i n f_{i}}}^{y_{s u p_{i}}} \int_{x_{i n f_{i}}}^{x_{s u p_{i}}}\left(-\frac{1}{\sigma_{x}}+\frac{\left(x-\mu_{x}\right)^{2}}{\sigma_{x}^{3}}\right) \phi(x, y) d x d y \\
& \frac{\partial^{2} l_{i}(\boldsymbol{\theta})}{\partial \alpha^{2}}=\frac{1}{\Phi_{i}(\cdot)} \int_{y_{\text {inf }}}^{y_{\text {sup }}} \int_{x_{i n f_{i}}}^{x_{\text {sup }}}\left(-\frac{1}{\sigma^{2}}+\frac{(y-\alpha-\beta x)^{2}}{\sigma^{4}}\right) \phi(x, y) d x d y \\
& -\frac{1}{\left(\Phi_{i}(\cdot)\right)^{2}}\left(\int_{y_{\text {inf }}}^{y_{\text {sup }_{i}}} \int_{x_{i n f_{i}}}^{x_{s u p_{i}}} \frac{y-\alpha-\beta x}{\sigma^{2}} \phi(x, y) d x d y\right)^{2}
\end{aligned}
$$




$$
\begin{aligned}
& \frac{\partial^{2} l_{i}(\boldsymbol{\theta})}{\partial \alpha \partial \beta}=\frac{1}{\Phi_{i}(\cdot)} \int_{y_{\text {inf }_{i}}}^{y_{\text {sup }_{i}}} \int_{x_{\text {inf }}}^{x_{\text {sup }_{i}}}\left(-\frac{x}{\sigma^{2}}+\frac{(y-\alpha-\beta x)^{2}}{\sigma^{4}}\right) \phi(x, y) d x d y \\
& -\frac{1}{\left(\Phi_{i}(\cdot)\right)^{2}}\left(\int_{y_{i n f_{i}}}^{y_{\text {sup }_{i}}} \int_{x_{\text {inf }}}^{x_{s u p_{i}}} \frac{y-\alpha-\beta x}{\sigma^{2}} \phi(x, y) d x d y\right. \\
& \left.\int_{y_{\text {inf }}}^{y_{\text {sup }}} \int_{x_{i n f_{i}}}^{x_{\text {sup }}} \frac{(y-\alpha-\beta x) x}{\sigma^{2}} \phi(x, y) d x d y\right) \\
& \frac{\partial^{2} l_{i}(\boldsymbol{\theta})}{\partial \alpha \partial \sigma}=\frac{1}{\Phi_{i}(\cdot)} \int_{y_{i n f_{i}}}^{y_{s u p_{i}}} \int_{x_{i n f_{i}}}^{x_{s u p_{i}}}\left(-\frac{3(y-\alpha-\beta x)}{\sigma^{3}}+\frac{(y-\alpha-\beta x)^{3}}{\sigma^{5}}\right) \phi(x, y) d x d y \\
& -\frac{1}{\left(\Phi_{i}(\cdot)\right)^{2}}\left(\int_{y_{i n f_{i}}}^{y_{s u p_{i}}} \int_{x_{i n f_{i}}}^{x_{s u p_{i}}} \frac{y-\alpha-\beta x}{\sigma^{2}} \phi(x, y) d x d y\right. \\
& \left.\int_{y_{i n f_{i}}}^{y_{\text {sup }_{i}}} \int_{x_{i n f_{i}}}^{x_{\text {sup }_{i}}}\left(-\frac{1}{\sigma}+\frac{(y-\alpha-\beta x)^{2}}{\sigma^{3}}\right) \phi(x, y) d x d y\right) \\
& \frac{\partial^{2} l_{i}(\boldsymbol{\theta})}{\partial \alpha \partial \mu_{x}}=\frac{1}{\Phi_{i}(\cdot)} \int_{y_{\text {inf }}}^{y_{\text {sup }}} \int_{x_{i n f_{i}}}^{x_{\text {sup }}}\left(\frac{(y-\alpha-\beta x)\left(x-\mu_{x}\right)}{\sigma^{2} \sigma_{x}^{2}}\right) \phi(x, y) d x d y \\
& -\frac{1}{\left(\Phi_{i}(\cdot)\right)^{2}}\left(\int_{y_{i n f_{i}}}^{y_{s u p_{i}}} \int_{x_{i n f_{i}}}^{x_{s u p_{i}}} \frac{y-\alpha-\beta x}{\sigma^{2}} \phi(x, y) d x d y\right.
\end{aligned}
$$

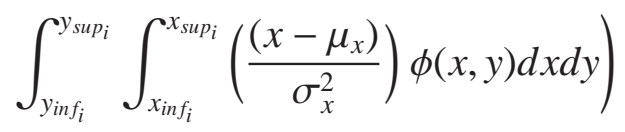

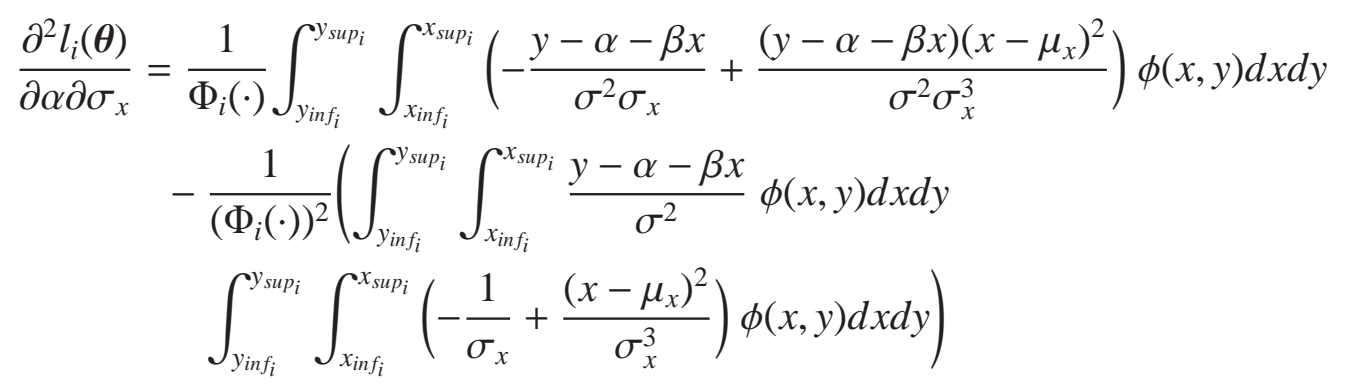




$$
\begin{aligned}
& \frac{\partial^{2} l_{i}(\boldsymbol{\theta})}{\partial \beta^{2}}=\frac{1}{\Phi_{i}(\cdot)} \int_{y_{\text {inf }}}^{y_{\text {sup }_{i}}} \int_{x_{\text {inf }}}^{x_{\text {sup }_{i}}}\left(-\frac{x^{2}}{\sigma^{2}}+\frac{(y-\alpha-\beta x)^{2} x^{2}}{\sigma^{4}}\right) \phi(x, y) d x d y \\
& -\frac{1}{\left(\Phi_{i}(\cdot)\right)^{2}}\left(\int_{y_{\text {inf }}}^{y_{\text {sup }_{i}}} \int_{x_{\text {inf }}}^{x_{\text {sup }_{i}}} \frac{(y-\alpha-\beta x) x}{\sigma^{2}} \phi(x, y) d x d y\right)^{2} \\
& \frac{\partial^{2} l_{i}(\boldsymbol{\theta})}{\partial \beta \partial \sigma}=\frac{1}{\Phi_{i}(\cdot)} \int_{y_{\text {inf }_{i}}}^{y_{\text {sup }_{i}}} \int_{x_{\text {inf }}}^{x_{\text {sup }_{i}}}\left(-\frac{3(y-\alpha-\beta x) x}{\sigma^{3}}+\frac{(y-\alpha-\beta x)^{3} x}{\sigma^{5}}\right) \phi(x, y) d x d y
\end{aligned}
$$

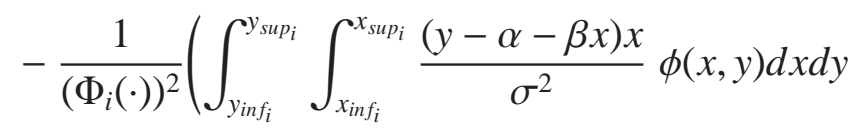

$$
\begin{aligned}
& \left.\int_{y_{\text {inf }}}^{y_{\text {sup }_{i}}} \int_{x_{\text {inf }}}^{x_{\text {sup }}}\left(-\frac{1}{\sigma}+\frac{(y-\alpha-\beta x)^{2}}{\sigma^{3}}\right) \phi(x, y) d x d y\right)
\end{aligned}
$$

$$
\begin{aligned}
& \frac{\partial^{2} l_{i}(\boldsymbol{\theta})}{\partial \beta \partial \mu_{x}}=\frac{1}{\Phi_{i}(\cdot)} \int_{y_{i n f_{i}}}^{y_{\text {sup }_{i}}} \int_{x_{\text {inf }}}^{x_{\text {sup }}}\left(\frac{(y-\alpha-\beta x) x\left(x-\mu_{x}\right)}{\sigma^{2} \sigma_{x}^{2}}\right) \phi(x, y) d x d y \\
& -\frac{1}{\left(\Phi_{i}(\cdot)\right)^{2}}\left(\int_{y_{\text {inf }}}^{y_{\text {sup }_{i}}} \int_{x_{\text {inf }}}^{x_{\text {sup }_{i}}} \frac{(y-\alpha-\beta x) x}{\sigma^{2}} \phi(x, y) d x d y\right. \\
& \left.\int_{y_{i n f_{i}}}^{y_{s u p_{i}}} \int_{x_{i n f_{i}}}^{x_{s u p_{i}}}\left(\frac{x-\mu_{x}}{\sigma_{x}^{2}}\right) \phi(x, y) d x d y\right)
\end{aligned}
$$

$$
\begin{aligned}
& \frac{\partial^{2} l_{i}(\boldsymbol{\theta})}{\partial \beta \partial \sigma_{x}}=\frac{1}{\Phi_{i}(\cdot)} \int_{y_{i n f_{i}}}^{y_{s u p_{i}}} \int_{x_{i n f_{i}}}^{x_{s u p_{i}}}\left(-\frac{(y-\alpha-\beta x) x}{\sigma^{2} \sigma_{x}}+\frac{(y-\alpha-\beta x) x\left(x-\mu_{x}\right)^{2}}{\sigma^{2} \sigma_{x}^{3}}\right) \phi(x, y) d x d y \\
& -\frac{1}{\left(\Phi_{i}(\cdot)\right)^{2}}\left(\int_{y_{\text {inf }}}^{y_{\text {sup }_{i}}} \int_{x_{\text {inf }}}^{x_{\text {sup }}} \frac{(y-\alpha-\beta x) x}{\sigma^{2}} \phi(x, y) d x d y\right. \\
& \left.\int_{y_{\text {inf }_{i}}}^{y_{\text {sup }_{i}}} \int_{x_{\text {inf }}}^{x_{\text {sup }_{i}}}\left(-\frac{1}{\sigma_{x}}+\frac{\left(x-\mu_{x}\right)^{2}}{\sigma_{x}^{3}}\right) \phi(x, y) d x d y\right)
\end{aligned}
$$




$$
\begin{aligned}
\frac{\partial^{2} l_{i}(\boldsymbol{\theta})}{\partial \sigma^{2}}= & \frac{1}{\Phi_{i}(\cdot)} \int_{y_{\text {inf }}}^{y_{\text {sup }}} \int_{x_{i n f_{i}}}^{x_{\text {sup }}}\left(\frac{1}{\sigma^{2}}-\frac{5(y-\alpha-\beta x)^{2}}{\sigma^{4}}+\frac{(y-\alpha-\beta x)^{4}}{\sigma^{6}}\right) \phi(x, y) d x d y \\
& -\frac{1}{\left(\Phi_{i}(\cdot)\right)^{2}}\left(\int_{y_{\text {inf }}}^{y_{\text {sup }}} \int_{x_{i n f_{i}}}^{x_{\text {sup }}}\left(-\frac{1}{\sigma}+\frac{(y-\alpha-\beta x)^{2}}{\sigma^{3}}\right) \phi(x, y) d x d y\right)^{2}
\end{aligned}
$$

$$
\begin{aligned}
\frac{\partial^{2} l_{i}(\boldsymbol{\theta})}{\partial \sigma \partial \mu_{x}}= & \frac{1}{\Phi_{i}(\cdot)} \int_{y_{\text {inf }}}^{y_{\text {sup }_{i}}} \int_{x_{\text {inf }}}^{x_{\text {sup }_{i}}}\left(-\frac{x-\mu_{x}}{\sigma \sigma_{x}^{3}}+\frac{(y-\alpha-\beta x)^{2}\left(x-\mu_{x}\right)}{\sigma^{3} \sigma_{x}^{2}}\right) \phi(x, y) d x d y \\
- & \frac{1}{\left(\Phi_{i}(\cdot)\right)^{2}}\left(\int_{y_{\text {inf }}}^{y_{\text {sup }_{i}}} \int_{x_{i n f_{i}}}^{x_{\text {sup }_{i}}}\left(-\frac{1}{\sigma}+\frac{(y-\alpha-\beta x)^{2}}{\sigma^{3}}\right) \phi(x, y) d x d y\right. \\
& \left.\int_{y_{\text {inf }}}^{y_{\text {su }_{i}}} \int_{x_{i n f_{i}}}^{x_{\text {sup }_{i}}} \frac{x-\mu_{x}}{\sigma_{x}^{2}} \phi(x, y) d x d y\right)
\end{aligned}
$$

$$
\begin{aligned}
\frac{\partial^{2} l_{i}(\boldsymbol{\theta})}{\partial \sigma \partial \sigma_{x}}= & \frac{1}{\Phi_{i}(\cdot)} \int_{y_{\text {inf }}}^{y_{\text {sup }}} \int_{x_{i n f_{i}}}^{x_{\text {sup }}}\left(\frac{1}{\sigma \sigma_{x}}-\frac{\left(x-\mu_{x}\right)^{2}}{\sigma \sigma_{x}^{3}}-\frac{(y-\alpha-\beta x)^{2}}{\sigma^{3} \sigma_{x}}+\right. \\
& \left.\frac{(y-\alpha-\beta x)^{2}\left(x-\mu_{x}\right)^{2}}{\sigma^{3} \sigma_{x}^{3}}\right) \phi(x, y) d x d y \\
- & \frac{1}{\left(\Phi_{i}(\cdot)\right)^{2}}\left(\int_{y_{\text {inf }}}^{y_{s u p_{i}}} \int_{x_{\text {inf }}}^{x_{\text {sup }}}\left(-\frac{1}{\sigma}+\frac{(y-\alpha-\beta x)^{2}}{\sigma^{3}}\right) \phi(x, y) d x d y\right. \\
& \left.\int_{y_{\text {inf }}}^{y_{\text {sup }}} \int_{x_{i n f_{i}}}^{x_{\text {sup }}}\left(-\frac{1}{\sigma_{x}}+\frac{\left(x-\mu_{x}\right)^{2}}{\sigma_{x}^{3}}\right) \phi(x, y) d x d y\right)
\end{aligned}
$$

$$
\begin{aligned}
\frac{\partial^{2} l_{i}(\boldsymbol{\theta})}{\partial \mu_{x}^{2}}= & \frac{1}{\Phi_{i}(\cdot)} \int_{y_{i n f_{i}}}^{y_{\text {sup }}} \int_{x_{i n f_{i}}}^{x_{\text {sup }}}\left(-\frac{1}{\sigma_{x}^{2}}+\frac{\left(x-\mu_{x}\right)^{2}}{\sigma_{x}^{4}}\right) \phi(x, y) d x d y \\
& -\frac{1}{\left(\Phi_{i}(\cdot)\right)^{2}}\left(\int_{y_{\text {inf }}}^{y_{s u p_{i}}} \int_{x_{i n f_{i}}}^{x_{s u p_{i}}}\left(\frac{x-\mu_{x}}{\sigma_{x}^{2}}\right) \phi(x, y) d x d y\right)^{2}
\end{aligned}
$$




$$
\begin{aligned}
& \frac{\partial^{2} l_{i}(\boldsymbol{\theta})}{\partial \mu_{x} \partial \sigma_{x}}=\frac{1}{\Phi_{i}(\cdot)} \int_{y_{i n f_{i}}}^{y_{s u p_{i}}} \int_{x_{i n f_{i}}}^{x_{s u p_{i}}}\left(-\frac{3\left(x-\mu_{x}\right)}{\sigma_{x}^{3}}+\frac{\left(x-\mu_{x}\right)^{3}}{\sigma_{x}^{5}}\right) \phi(x, y) d x d y \\
& -\frac{1}{\left(\Phi_{i}(\cdot)\right)^{2}}\left(\int_{y_{i n f_{i}}}^{y_{s u p_{i}}} \int_{x_{i n f_{i}}}^{x_{s u p_{i}}}\left(\frac{x-\mu_{x}}{\sigma_{x}^{2}}\right) \phi(x, y) d x d y\right. \\
& \left.\int_{y_{\text {inf }}}^{y_{\text {sup }}} \int_{x_{i n f_{i}}}^{x_{\text {sup }} i}\left(-\frac{1}{\sigma_{x}}+\frac{\left(x-\mu_{x}\right)^{2}}{\sigma_{x}^{3}}\right) \phi(x, y) d x d y\right) \\
& \frac{\partial^{2} l_{i}(\boldsymbol{\theta})}{\partial \sigma_{x}^{2}}=\frac{1}{\Phi_{i}(\cdot)} \int_{y_{i n f_{i}}}^{y_{s u p_{i}}} \int_{x_{i n f_{i}}}^{x_{s u p_{i}}}\left(\frac{1}{\sigma_{x}^{2}}-\frac{5\left(x-\mu_{x}\right)^{2}}{\sigma_{x}^{4}}+\frac{\left(x-\mu_{x}\right)^{4}}{\sigma_{x}^{6}}\right) \phi(x, y) d x d y \\
& -\frac{1}{\left(\Phi_{i}(\cdot)\right)^{2}}\left(\int_{y_{\text {infi }}}^{y_{\text {sup }}} \int_{x_{i n f_{i}}}^{x_{\text {sup }}}\left(-\frac{1}{\sigma_{x}}+\frac{\left(x-\mu_{x}\right)^{2}}{\sigma_{x}^{3}}\right) \phi(x, y) d x d y\right)^{2}
\end{aligned}
$$





\section{Referências Bibliográficas}

Aitkin, M. (1981). A note on the regression-analysis of censored-data. Technometrics 23, 161-163.

Amemiya, T. (1973). Regression-analysis when dependent variable is truncated normal. Econometrica 41, 997-1016.

Austin, P. C. and Brunner, L. J. (2003). Type I error inflation in the presence of a ceiling effect. American Statistician 57, 97-104.

Austin, P. C. and Hoch, J. S. (2004). Estimating linear regression models in the presence of a censored independent variable. Statistics in Medicine 23, 411429.

Casella, G. and Berger, R. (1990). Statistical Inference. Belmont, Wadsworth Inc.

Chatterjee, S. and McLeish, D. L. (1986). Fitting linear-regression models to censored-data by least-squares and maximum-likelihood methods. Communications in Statistics-Theory and Methods 15, 3227-3243.

Dempster, A. P., Laird, N. M., and Rubin, D. B. (1977). Maximum likelihood from incomplete data via em algorithm. Journal of the Royal Statistical Society Series B-Methodological 39, 1-38.

Duarte, J. L. (2007). A utilização da resposta auditiva de estado estável para estimar limiares em indivíduos com perda neurossensorial. Dissertação de Mestrado, Faculdade de Odontologia de Bauru da Universidade de São Paulo. 
Glasser, M. (1965). Regression analysis with dependent variable censored. Biometrics 21, 300-307.

Gomez, G., Espinal, A., and Lagakos, S. W. (2003). Inference for a linear regression model with an interval-censored covariate. Statistics in Medicine 22, 409-425.

Haas, C. N. and Jacangelo, J. G. (1993). Development of regression-models withn below-detection data. Journal of Environmental Engineering-Asce 119, 214230.

Klein, J. P. and Moeschberger, M. L. (1997). Survival analysis: techniques for censored and truncated data. New York, Springer-Verlag.

Lachenbruch, P. A., Clements, P. J., and He, W. Z. (1995). On encoding values for data recorded as X > C. Biometrical Journal 37, 855-867.

Lins, O. G. and Picton, T. W. (1995). Auditory steady-state responses to multiple simultaneous stimuli. Evoked Potentials-Electroencephalography and Clinical Neurophysiology 96, 420-432.

Maplesoft (2004). Maple 9.5 Getting Started Guide. Toronto, Maplesoft, a division of Waterloo Maple Inc.

Neter, J., Kutner, M., Nachtsheim, C., and Wasserman, W. (1996). Applied Linear Statistical Models. 4th edition, New York, WCB/McGraw-Hill.

Northern, J. L. and Downs, M. P. (2002). Hearing in Children. 5th edition, Philadelphia, Lippincott Williams \& Wilkins.

Ren, J. J. (2003). Goodness of fit tests with interval censored data. Scandinavian Journal of Statistics 30, 211-226.

Russo, I. C. P. (1999). Acústica e Psicoacústica Aplicadas à Fonoaudiologia. 2a. edição, São Paulo, Editora Lovise Ltda. 
SAS Institute Inc (2004). SAS OnlineDoc $₫ 9$ 9.1.3. Cary, North Carolina, SAS Institute Inc.

Schmee, J. and Hahn, G. J. (1979). Simple method for regression-analysis with censored data. Technometrics 21, 417-432.

Schneider, H. (1986). Truncated and Censored Samples from Normal populations. New York, Marcel Dekker.

Sharma, M., Thomson, N. R., and McBean, E. A. (1995). Linear-regression analyses with censored-data - estimation of pah washout ratios and dry deposition velocities to a snow surface. Canadian Journal of Civil Engineering 22, 819833.

Thisted, R. A. (1988). Elements of Statistical Computing. New York, Chapman $\&$ Hall.

Thompson, M. L. and Nelson, K. P. (2003). Linear regression with type I intervaland left-censored response data. Environmental and Ecological Statistics 10, 221-230.

Tobin, J. (1958). Estimation of relationships for limited dependent-variables. Econometrica 26, 24-36.

Topp, R. and Gomez, G. (2004). Residual analysis in linear regression models with an interval-censored covariate. Statistics in Medicine 23, 3377-3391.

Turnbull, B. W. (1976). Empirical distribution function with arbitrarily grouped, censored and truncated data. Journal of the Royal Statistical Society Series B-Methodological 38, 290-295. 